\title{
An Analytical Model for Wedge-Shaped Acoustic Arrays
}

\author{
Falk-Martin Hoffmann ${ }^{\mathrm{a}, *}$, Earl G. Williams ${ }^{\mathrm{b}}$, Filippo Maria Fazi $^{\mathrm{a}}$, Simone Fontana ${ }^{\mathrm{c}}$ \\ ${ }^{a}$ Institute of Sound and Vibration Research, University of Southampton, University Road, Southampton, SO17 1BJ, United Kingdom \\ ${ }^{b}$ Naval Research Laboratory, Washington, D.C., USA \\ ${ }^{c}$ HUAWEI European Research Center, Riesstrasse 25 C3.0G, 80992 Munich, Germany
}

\begin{abstract}
This work presents the derivation of a 3D sound field model for the pressure field radiated from loudspeaker arrays in acoustic wedge spaces. These wedge arrays are described by their normal velocity profile at a given boundary. Using this model, a suitable beamforming technique with two different beam patterns is derived based on a mode-matching approach. The model is then used to study the beamforming performance as a function of frequency and distance from the array by analysing the Arc Radial Functions introduced in this work. The theory is tested on the basis of simulation results for different wedges and validated through holographic measurements made with a hemi-cylindrical array. It is found that for a large distance, a far-field approximation of the model can be used to drive the array, whereas for a short distance, the general model must be applied to yield accurate results.
\end{abstract}

Keywords: wedge space, wedge array, inverse problem, arc radial function, beamforming, near-field model PACS: 43.20.Rz, 43.38.Hz, 43.40.Sk, 43.60.Fg

\section{Introduction}

Loudspeaker arrays have become established means for sound field control applications in various types of environments. Until recently, research was dominated by three principal array configurations: linear arrays (e.g. for line arrays in public address (PA) applications, 2D Wave Field Synthesis (WFS) etc.) [1,2], spherical arrays [3-5] and cylindrical/circular arrays [6-8]. Linear arrays are probably the most visible/available to the public, ranging from PA applications to personal audio systems in the form of sound bars. Cylindrical/circular and spherical arrays are often found in the form of large installations for Wave Field Synthesis (WFS) or Higher Order Ambisonics (HOA) systems [3-5, 9-12], but also in compact form for sound sources with directivity control [6, 7, 13-16].

The term 'sound field control' can be interpreted in a relatively wide sense. The most classic form is probably the generation of a sound field in a dedicated reproduction area with holophony as the objective [2, 3, 8]. Acoustic contrast control and pressure matching, as well as acoustic beamforming are also forms of sound field control [6, 17-20]. All these techniques can (but do not have to) be derived on the basis of wave propagation models, between which the physical accuracy may vary for a given environment. Two major assumptions are typically made to simplify such models:

\footnotetext{
* Corresponding Author

Email addresses: F.Hoffmann@soton.ac.uk (Falk-Martin Hoffmann), Earl.Williams@nrl.navy.mil (Earl G. Williams), F.Fazi@isvr.soton.ac.uk (Filippo Maria Fazi), Simone.Fontana@huawei.com (Simone Fontana)
}

first, that the array is located in an infinite propagation space and, second, that the transducers are acoustically transparent (i.e. free-field conditions). Both assumptions are typically not valid in practice, thus effecting a less than ideal performance of the real system. A good amount of work has been published on the modelling of the diffraction as it occurs on spherical and cylindrical structures housing transducer arrays [13, 21-25], but also modelling of the reflections caused by the environment has been proposed to improve sound field control in reflective environments $[26,27]$. Pomberger et al. described the diffraction caused by a rigid sphere in a propagation space with rigid boundary conditions at two polar angles. [28, 29]. A special case of practical importance receiving so far only limited attention is a model for loudspeaker arrays radiating into the half-space.

An initial attempt to model a loudspeaker array arranged on an infinite, planar and rigid baffle was presented in [30]. The proposed hemi-cylindrical arrays were designed to be mounted on an infinite planar baffle (e.g. a wall) and perform beamforming into the half-space. The proposed beamforming method is based on a far-field wave propagation model and therefore may not model the near field of the array accurately. It is worth noting that the half-space is in fact a special case of a wedge-shaped propagation space, and thus the hemi-cylindrical array is a special case of a wedge-shaped array. The acoustic environment where such wedge arrays become relevant ranges from in-room applications (e.g. sound bars, PA systems, etc.) to cars or other means of transport, where the propagation space is inherently limited and can be described specifically or 
more abstractly as a wedge.

This work presents the derivation of a beamforming technique for loudspeaker arrays in infinite wedge propagation spaces [31]. The wave field models and basis functions for such propagation spaces have already found frequent use in the field of underwater acoustics [32, 33] and a work on Nearfield Acoustical Holography (NAH) in wedge spaces was recently presented by the authors in [34]. Using the existing models, two types of control filters for wedge arrays are proposed and tested in both simulations and experimental work.

The remainder of this work is organised as follows. The second section reviews the model for acoustic radiation with given boundary conditions in wedge propagation spaces and develops a general expression for the particle velocity produced by an array of loudspeakers located on that boundary. The third section applies the developed array radiation model to solve the inverse problem and calculate the loudspeaker driving functions for a desired pressure field through a mode-matching approach [18]. In that context, the low frequency behaviour and the occurrence of spatial aliasing caused by a finite number of loudspeakers are investigated. In Section four, the two types of beam patterns that are used for the simulations and measurements are introduced. Section five presents and discusses the simulations of radiated beam patterns across frequency for two examples of wedge arrays, where one of them serves to introduce the prototype used for the experiments. The obtained experimental results are described and discussed in Section six, and the final section summarises the findings and gives an outlook on upcoming research.

\section{Sound Field Model}

This work uses the sound field model for the radiation from a velocity distribution on a curved baffle $V$ around the origin of an infinite wedge propagation space, as recently presented by the authors in [34]. Note that all considerations are made for the (quasi-)stationary case, where the factor $\mathrm{e}^{-\mathrm{i} \omega t}$ and the different quantities' dependency on $\omega$ was omitted for reasons of brevity.

The geometrical description of the infinite wedge is based on the cylindrical coordinate system, as given in Figure 1. A projection of the propagation space onto the $x y$-plane with the added curved baffle $V$ holding the loudspeaker array is shown in Figure 2.

The propagation space $W$ is uniquely defined as

$$
W:=\left\{\mathbf{r}=(r, \phi, z)^{T}: r \geq r_{S}, \phi \in\left[\phi_{1}, \phi_{2}\right], z \in \mathbb{R}\right\},
$$

where $r_{S}$ is the radius of the curved baffle around the $z$-axis. The wedge angle $\kappa=\phi_{2}-\phi_{1}$ can take any value from the interval $[0,2 \pi]$. The boundaries of the propagation space comprise the curved baffle

$$
V:=\left\{\mathbf{r}: r=r_{S}, \phi \in\left[\phi_{1}, \phi_{2}\right], z \in \mathbb{R}\right\}
$$

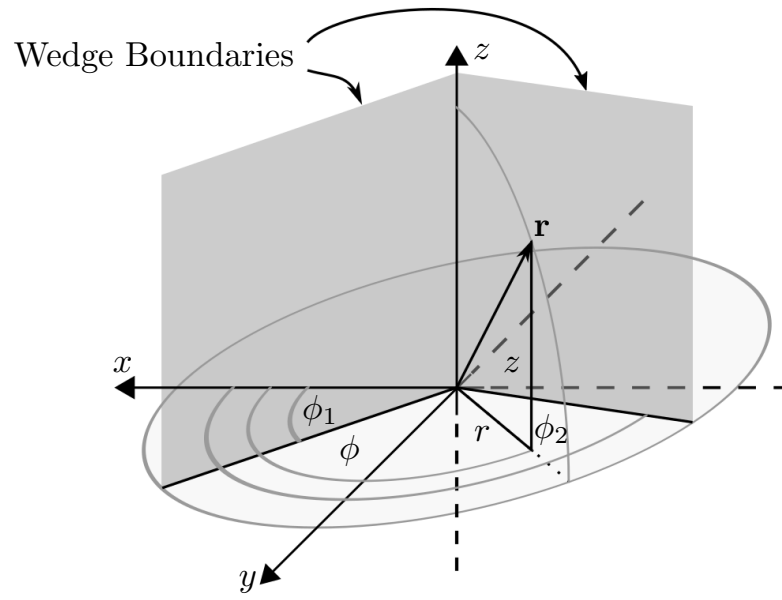

Figure 1: Propagation space with wedge boundaries described in cylindrical coordinates.

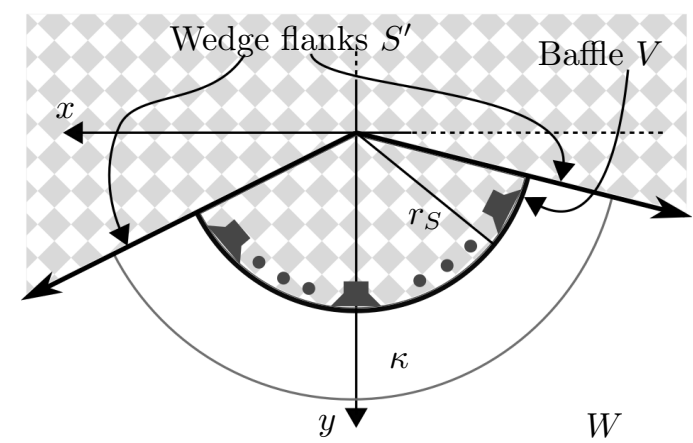

Figure 2: Wedge boundaries projected onto the $x y$-plane with the loudspeaker array mounted on the curved baffle.

and the wedge's flanks

$$
S^{\prime}:=\left\{\mathbf{r}: r>r_{S}, \phi=\phi_{1}, \phi_{2}, z \in \mathbb{R}\right\} .
$$

In the case of wedge arrays, all loudspeakers, i.e. all active acoustic sources, are located on $V$. Accordingly, the acoustic velocity on $V$ is defined by the driving functions and the physical dimensions of the transducers. Without loss of generality but to simplify notation, it is assumed in the remainder of this work that $\phi_{1}=0$ and $\phi_{2}=\kappa$.

The following subsection describes the mathematical model for the pressure radiated by a wedge array.

\subsection{General Pressure Model}

The general solution for the pressure field radiated from a given velocity distribution on $V$ inside an infinite wedge with rigid boundaries was presented in [34] and is given by the expression

$$
p(r, \phi, z)=\sum_{n=0}^{\infty} \Phi_{n}(\phi) \int_{-\infty}^{\infty} \Gamma_{n}\left(k_{z}, r\right) V_{n}\left(k_{z}, r_{S}\right) Z\left(k_{z} z\right) \mathrm{d} k_{z}
$$


with

$$
\begin{aligned}
\Gamma_{n}\left(k_{z}, r\right) & =\mathrm{i} \rho_{0} c k \frac{H_{\frac{n \pi}{\kappa}}\left(k_{r} r\right)}{k_{r} H_{\frac{n \pi}{\kappa}}^{\prime}\left(k_{r} r_{S}\right)} \\
\Phi_{n}(\phi) & =\nu_{n} \cos \left(\frac{n \pi}{\kappa} \phi\right), \\
Z\left(k_{z} z\right) & =\frac{\mathrm{e}^{\mathrm{i} k_{z} z}}{\sqrt{2 \pi}},
\end{aligned}
$$

where

$$
k_{r}=\sqrt{k^{2}-k_{z}^{2}} \quad \text { and } \quad \nu_{n}=\sqrt{\frac{2-\delta_{n}}{\kappa}},
$$

$\kappa$ is the wedge angle, $k$ represents the acoustic wave number and $\delta_{n}$ denotes the Kronecker Delta. $H_{\frac{n \pi}{\kappa}}(x)$ refers to the Hankel function of the first kind ${ }^{1}$ of order $\frac{n \pi}{\kappa}$, where the latter is exclusively integer only for $\kappa=\pi$, and $H_{\frac{n \pi}{\kappa}}^{\prime}(x)$ denotes the derivative. The angular basis functions $\Phi_{n}^{\kappa}(\phi)$ and the axial basis functions $Z\left(k_{z} z\right)$ are the elements of two Total Orthonormal Sets (TOS) [35]. Their product creates a two-dimensional TOS. $\Gamma_{n}\left(k_{z}, r\right)$ denotes the corresponding radial functions for the radiation problem in the wedge space [34], where the dependency on $\kappa$ and $r_{S}$ was omitted for the sake of brevity. The same applies for the angular basis functions' dependency on $\kappa$ and $\phi_{1}$.

The spatial velocity spectrum $V_{n}\left(k_{z}, r_{S}\right)$ in Eq. (2) is calculated as the spatial transform of the normal velocity (or vibration) profile $v\left(r_{S}, \phi, z\right)$ describing the particle displacement in the normal direction on the boundary $V$ [34]

$$
\begin{aligned}
V_{n}\left(k_{z}, r_{S}\right) & =\mathcal{W}\left[v\left(r_{S}, \phi, z\right)\right] \\
& =\int_{0}^{\kappa} \int_{-\infty}^{\infty} v\left(r_{S}, \phi, z\right) \Phi_{n}(\phi) Z^{*}\left(k_{z} z\right) \mathrm{d} z \mathrm{~d} \phi,
\end{aligned}
$$

where $(\cdot)^{*}$ denotes the complex conjugate and the operator $\mathcal{W}$ abbreviates the spatial transform. Inversely, $v\left(r_{S}, \phi, z\right)$ is obtained from $V_{n}\left(k_{z}, r_{S}\right)$ via the inverse operator

$$
\begin{aligned}
v\left(r_{S}, \phi, z\right) & =\mathcal{W}^{-1}\left[V_{n}\left(k_{z}, r_{S}\right)\right] \\
& =\sum_{n=0}^{\infty} \int_{-\infty}^{\infty} V_{n}\left(k_{z}, r_{S}\right) \Phi_{n}(\phi) Z\left(k_{z} z\right) \mathrm{d} k_{z} .
\end{aligned}
$$

Equations (6) and (7) define a transformation pair that may be regarded as a generalised Fourier transform.

With the general sound field model at hand, the sound field control problem can now be theoretically solved by taking a mode-matching approach that yields a relation between the pressure spectrum

$$
P_{n}\left(k_{z}, r\right)=\mathcal{W}[p(r, \phi, z)],
$$

of a desired pressure field $p(r, \phi, z)$ at the radial coordinate $r$ and the velocity spectrum $V_{n}\left(k_{z}, r_{S}\right)$ on $V$.

\footnotetext{
${ }^{1}$ The superscript ${ }^{(1)}$ commonly used to distinguish the Hankel functions of the first from that of the second kind is omitted for reasons of brevity.
}

\subsection{The General Solution to the Inverse Problem}

Finding the required vibration profile $v$ on $V$ that yields a specific target pressure field $p$ is an inverse problem that needs solving.

Applying the inverse operator $\mathcal{W}^{-1}[\cdot]$ defined in Eq. (7) to both sides of Eq. (2) yields

$$
P_{n}\left(k_{z}, r\right)=\Gamma_{n}\left(k_{z}, r\right) V_{n}\left(k_{z}, r_{S}\right) .
$$

Equation (9) can be transformed into an expression for the (normal) velocity spectrum

$$
V_{n}\left(k_{z}, r_{S}\right)=\frac{1}{\Gamma_{n}\left(k_{z}, r\right)} P_{n}\left(k_{z}, r\right) .
$$

The required normal velocity on the boundary $V$ can then be obtained by replacing the velocity spectrum in Eq. (7) with the result in Eq. (10). From this it follows that, in general, an infinitely long (along the $z$-axis) vibrating baffle is required for the reconstruction of a desired pressure field $p(r, \phi, z)$ that satisfies the homogeneous Helmholtz equation in $W$.

Controlling the vibration profile on the entire surface $V$ is impractical. Therefore this work focusses on sound field control on an arbitrary plane $z=z_{B}$ using an array with a finite number of loudspeakers arranged on an arc. This is henceforth referred to as the wedge array. The radiated sound field of course still satisfies the homogeneous Helmholtz equation in $W$, despite the finite extension of the vibrating aperture. Before presenting the methods to identify the individual loudspeakers' driving functions, a general expression for the velocity spectrum of arbitrary wedge arrays is defined in the next subsection.

\subsection{Velocity Spectrum of an Arbitrary Wedge Array}

Let a wedge array be composed of $L$ loudspeakers. Each speaker unit is described by the location of its geometrical centre $\left(r_{S}, \phi_{l}, z_{l}\right)^{T} \in V$ and its diaphragm's velocity profile $v_{l}\left(r_{S}, \phi, z\right)$. The index $l$ is used to refer to the $l$ th speaker in the array. In general, the corresponding velocity spectra, $V_{n}^{l}\left(k_{z}\right)$, are determined with the operator $\mathcal{W}$ defined in Eq. (6).

It shall be assumed that the diaphragm is a rectangular piston [34] of angular width $\Theta$ and vertical length $B$. The corresponding velocity profile of the $l$ th loudspeaker is then given by

$$
v_{l}\left(r_{S}, \phi, z\right)=\Pi\left(\frac{\phi-\phi_{l}}{\Theta}\right) \Pi\left(\frac{z-z_{l}}{B}\right) q_{l},
$$

where $\Pi(\cdot)$ denotes the rectangular function and $q_{l}$ denotes the driving function. Applying $\mathcal{W}[\cdot]$ to the above equation yields the diaphragm's velocity spectrum (compare [34])

$$
V_{n}^{l}\left(k_{z}\right)=V_{n}^{D}\left(k_{z}\right) \Phi_{n}\left(\phi_{l}\right) Z^{*}\left(k_{z} z_{l}\right) q_{l}
$$

with

$$
V_{n}^{D}\left(k_{z}\right)=\Theta \operatorname{sinc}\left(\frac{n \pi}{\kappa} \frac{\Theta}{2}\right) B \operatorname{sinc}\left(k_{z} \frac{B}{2}\right)
$$


where $\operatorname{sinc}(x)=\frac{\sin x}{x}$. The explicit dependency of $V_{n}^{l}\left(k_{z}\right)$ on $r_{S}$ has been omitted for the sake of brevity. The expression in Eq. (11) was deliberately separated into a factor defining the shape of the diaphragm, $V_{n}^{D}\left(k_{z}\right)$, and two factors defining its position. Note that $V_{n}^{D}\left(k_{z}\right)=1$ for a point source, which shall also be considered later on. Although the form of Eq. (11) may suggest otherwise, the general shift property is not valid for the transform $\mathcal{W}[\cdot]$.

For the velocity spectrum of the full wedge array it is convenient to assume that all loudspeakers have the same shape, i.e. $V_{n}^{D}\left(k_{z}\right)$ is equal for all $L$ loudspeakers. This step is not strictly necessary in general, but it serves to simplify notation. Assuming linearity, the superposition principle enables the description of the overall velocity spectrum, $V_{n}\left(k_{z}, r_{S}\right)$, as the linear combination of the loudspeakers' velocity spectra $V_{n}^{l}\left(k_{z}\right)$

$$
V_{n}\left(k_{z}, r_{S}\right)=V_{n}^{D}\left(k_{z}\right) \sum_{l=1}^{L} q_{l} \Phi_{n}\left(\phi_{l}\right) Z^{*}\left(k_{z} z_{l}\right) .
$$

The triple $\left(q_{l}, \phi_{l}, z_{l}\right)$ defines each loudspeaker's driving function, angular position and height, respectively. The specific form of Eq. (13) will prove useful in Section 3.

The result for $V_{n}\left(k_{z}, r_{S}\right)$ in Eq. (13) can now be used to first formulate the array-specific forward problem and then solve the corresponding inverse problem in the modal domain.

\subsection{The Array-Specific Inverse Problem}

Replacing $V_{n}\left(k_{z}, r_{S}\right)$ in Eq. (9) with the expression in Eq. (13) provides the relation of the array's driving functions, $q_{l}$, to the radiated pressure spectrum

$$
P_{n}\left(k_{z}, r\right)=\Gamma_{n}\left(k_{z}, r\right) V_{n}^{D}\left(k_{z}\right) \sum_{l=1}^{L} q_{l} \Phi_{n}\left(\phi_{l}\right) Z^{*}\left(k_{z} z_{l}\right)
$$

for $n \in \mathbb{N}_{0}$ and $k_{z} \in \mathbb{R}$. The inverse problem of Eq. (14) is highly overdetermined since the $L$ driving functions $q_{l}$ are to control infinitely many functions $P_{n}\left(k_{z}, r\right)$ defined in the $k_{z}$-domain. Such a system may have a solution, but in general it cannot be solved exactly, unless one were to truncate the range of $n$ and to sample and truncate the range of $k_{z}$; In that case one would obtain an approximation of the target pressure.

The modes corresponding to the indices $\left(n, k_{z}\right)$ that fall into the region outside the "radiation circle", defined by $k \leq \sqrt{k_{z}^{2}+\left(\frac{n \pi}{\kappa r_{S}}\right)^{2}}$ [21], are evanescent in their nature. Therefore their corresponding magnitude $\left|P_{n}\left(k_{z}, r\right)\right|$ is subject to a steep decay as the radial coordinate $r$ increases. This physical effect is mathematically described in Eq. (14) by the magnitude of the radial functions $\Gamma_{n}\left(k_{z}, r\right)$. As a consequence, mostly modes from within or marginally outside the "radiation circle" need to be considered for a good approximation of the desired sound field. Therefore, limiting the range of $n$ and $k_{z}$ may not have a severely aggravating effect on the inverse solution. However, the uncountable nature of the domain of definition for the functions $P_{n}\left(k_{z}\right)$ necessitates sampling the latter along the $k_{z}$-axis to calculate a numerical solution.

The considerations made in this section indicate that full control of the sound field inside the infinite wedge cannot be achieved with a finite set of transducers arranged within a finite area on $V$. This situation changes when the objective is simplified to controlling the sound field on an arc with radius $r_{B}$ in the plane $z=z_{B}$ with loudspeakers arranged on the intersection of $V$ with the plane $z=z_{S}$. In that case, the driving functions $q_{l}$ can be identified using a mode-matching approach, which is shown in the following section.

\section{Loudspeaker Driving Functions}

\subsection{Diaphragm Velocity as a Function of Driving Voltage}

The driving functions derived in this section relate directly to the individual loudspeaker's velocity. When operating a classical electrodynamic transducer above its resonance frequency as a loudspeaker connected to an (ideal) voltage source, the applied voltage does not generate a proportional velocity $v$ of the diaphragm but a proportional acceleration $\dot{v}$ instead (compare [36] p. 61, Figure 5.7). In the frequency domain, the relation between voltage $u$ and velocity $v$ is therefore dominated by

$$
\frac{u(\omega)}{-\mathrm{i} \omega} \sim v(\omega)
$$

which corresponds to an integration in the time domain. It is therefore necessary to amend the driving signals $q_{l}(\omega)$ appropriately when operating an array built from electrodynamic loudspeakers through a conventional power amplifier. Note that Eq. (15) is only an approximation for the case when the electrical impedance is predominantly resistive and the mechanical impedance is dominated by the moving mass.

\subsection{Model for the Mode-Matching Approach}

From the completeness relation of TOSs [35], it follows that an arbitrary target pressure field on the arc

$$
\Psi:=\left\{\mathbf{r}: r=r_{B}, \phi \in[0, \kappa], z=z_{B}\right\}
$$

can be expressed as the infinite series expansion

$$
p\left(r_{B}, \phi, z_{B}\right)=\sum_{n=0}^{\infty} P_{n} \Phi_{n}(\phi) .
$$

To find a solution for the $L<\infty$ driving functions $q_{l}$ dependent on the target coefficients $P_{n}$, the driving functions need to first be expressed as a finite series expansion

$$
q_{l}=\sum_{m=0}^{L-1} Q_{m} \Phi_{m}\left(\phi_{l}\right), \forall l \in[1, L]
$$


The above expression poses a linear system of equations. Due to linear independence of $\Phi_{n}(\phi)$ for different orders, the system matrix has full rank and a unique solution for the coefficients $Q_{m}$ exists, if all $L$ angles $\phi_{l}$ are distinct.

Applying Eq. (17) in Eq. (13) and rearranging the double sum yields the expression

$$
V_{n}\left(k_{z}, r_{S}\right)=V_{n}^{D}\left(k_{z}\right) \sum_{m=0}^{L-1} \sum_{l=1}^{L} \Phi_{m}\left(\phi_{l}\right) \Phi_{n}\left(\phi_{l}\right) Z^{*}\left(k_{z} z_{l}\right) Q_{m}
$$

Equation (18) describes the modal relations between the velocity spectrum $V_{n}\left(k_{z}\right)$ and the coefficients $Q_{m}$ for the most general case. It is shown in the next section that a convenient choice of the parameters $\left(\phi_{l}, z_{l}\right)$ is useful for the mode-matching approach.

\subsection{Loudspeaker Positioning}

The loudspeaker positions are now chosen as uniformly distributed along the intersection of $V$ and $z=z_{S}$, i.e.

$$
\phi_{l}=\frac{\kappa}{L}\left(l-\frac{1}{2}\right) \quad \text { and } \quad z_{l}=z_{S}, \forall l \in[1 \ldots L] .
$$

This particular choice for $\phi_{l}$ simplifies the expression in Eq. (18) so that

$$
\begin{aligned}
V_{n}\left(k_{z}, r_{S}\right) & =V_{n}^{D}\left(k_{z}\right) \sum_{m=0}^{L-1} Z^{*}\left(k_{z} z_{S}\right) \frac{L}{\kappa} A_{n, m}, \\
\text { with } A_{n, m} & =\frac{\kappa}{L} \sum_{l=1}^{L} \Phi_{m}\left(\phi_{l}\right) \Phi_{n}\left(\phi_{l}\right) .
\end{aligned}
$$

Based on the properties of the Discrete Cosine Transform [37], it can be shown that the orthogonality matrix, also referred to as the spatial aliasing matrix, is given by the coefficients

$$
A_{n, m}=\frac{\sqrt{2-\delta_{n}} \sqrt{2-\delta_{m}}}{2} \sum_{u=0}^{\infty}(-1)^{u}\left[\delta_{n-m-2 L u}+\delta_{n+m-2 L u}\right]
$$

Using the result of Eq. (20) and replacing the result for $V_{n}\left(k_{z}\right)$ in Eq. (2) yields the expression

$$
\begin{gathered}
p\left(r_{B}, \phi, z_{B}\right)=\frac{L}{\kappa} \sum_{n=0}^{\infty} \sum_{m=0}^{L-1} \Phi_{n}(\phi) A_{n, m} Q_{m} \\
\cdot \int_{-\infty}^{\infty} \Gamma_{n}\left(k_{z}, r_{B}\right) V_{n}^{D}\left(k_{z}\right) Z^{*}\left(k_{z} z_{S}\right) Z\left(k_{z} z_{B}\right) \mathrm{d} k_{z} .
\end{gathered}
$$

In order to further simplify this result and understand why it was convenient for all loudspeakers to have the same diaphragm and the chosen positions, it is necessary to take a closer look at the coefficients $A_{n, m}$.

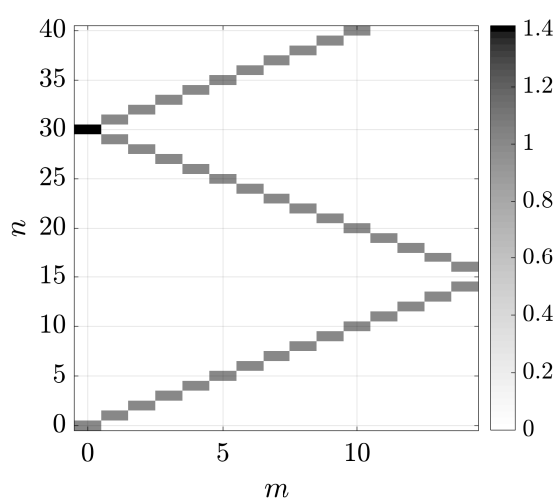

Figure 3: Visualisation of the magnitude of the orthogonality matrix, $A_{n, m}$, for $L=15$.

\subsection{Orthonormality and Aliasing}

An example of the coefficients specified by Eq. (22) for $L=15, n=0 \ldots 40$ and $m=0 \ldots 14=L-1$ is presented in Figure 3. It shows that the chosen positions of the $L$ loudspeakers preserve a perfect orthonormality relation of the modes $\Phi_{n}(\phi)$ for $n=0 \ldots L-1$. It also shows, however, that higher orders $n$ are subject to aliasing from the orders $m=0 \ldots L-1$, which can be predicted from Eq. (22). Note that both the pattern and thus also the expression on the right hand side of Eq. (22) would likely have been much more complicated if not for the assumption that all loudspeakers are identical as specified in Section 2.3.

The analysis of the coefficients $A_{n, m}$ shows that the finite number of loudspeakers still excites an infinite number of angular modes in the pressure field, even though an attempt is made to control the lowest $L$ modes $\Phi_{n}(\phi)$. The excitation of modes of higher order then manifests itself in the form of spatial aliasing artefacts in the reproduced field. These are well-known phenomena in the field of circular and spherical transducer arrays [7, 10, 11, 24, 38-42].

As discussed in Section 2.3, not all angular modes actually propagate to the far field. Those that are evanescent decay rapidly away from the surface of the array, making their contribution to the pressure on $\Psi$ potentially insignificant for large $r$. This is further investigated in the subsequent section.

\subsection{The Arc Radial Functions}

The integral and the leading factor of Equation (23) can be unified to form a new set of quantities that shall be denoted as Arc Radial Functions (ARFs)

$$
\Gamma_{n}^{\Psi}\left(r_{B}, z_{B}\right)=\frac{L}{\kappa 2 \pi} \int_{-\infty}^{\infty} \Gamma_{n}\left(k_{z}, r_{B}\right) V_{n}^{D}\left(k_{z}\right) \mathrm{e}^{\mathrm{i} k_{z}\left(z_{B}-z_{S}\right)} \mathrm{d} k_{z} .
$$

Together they describe how the modes excited by the specific set of driving functions $q_{l}$ propagate to the arc $\Psi$. The expression for the pressure on the arc simplifies to the 
infinite expansion of the weighted modes $\Phi_{n}(\phi)$ and reads as

$$
p\left(r_{B}, \phi, z_{B}\right)=\sum_{n=0}^{\infty} \sum_{m=0}^{L-1} \Phi_{n}(\phi) Q_{m} A_{n, m} \Gamma_{n}^{\Psi}\left(r_{B}, z_{B}\right) .
$$

The coefficients $Q_{m}$ do not directly determine the modal strength of $\Phi_{n}(\phi)$ for $n=m$. Instead, the ARFs describe how the $n$th angular mode excited on the source arc couples to the corresponding mode on the control arc $\Psi$. Which angular modes on the source arc get excited by the source distribution is determined by the coefficients $A_{n, m}$.

Figure 4 shows the development of the ARFs for different combinations of $r_{S}$ and $r_{B}$ with $\kappa=\frac{\pi}{2}$ for the velocity spectrum $V_{n}^{D}\left(k_{z}\right)$ of a single point source ${ }^{2}$. The propagation space associated with $\kappa=\frac{\pi}{2}$ corresponds to an infinitely tall, square corner. It can be observed that, as frequency increases, eventually all depicted orders propagate equally well. Naturally the overall attenuation depends on the propagation distance $r_{B}$.

At low frequencies $(f<3 \mathrm{kHz})$ it can be seen that the higher its order the more a mode gets attenuated due to evanescence. The frequencies $f_{n}^{C}=\frac{n \pi c}{2 \pi \kappa r_{S}}$ indicate where a mode of order $n$ changes from propagating to evanescent behaviour, as can be seen in Fig. 4d. The expression for the edge frequency $f_{n}^{C}$ was derived from the $n=k r$ rule that determines the transition from propagating to evanescent components [21, 43]. For the wedge, the rule becomes $\frac{n \pi}{\kappa}=k r$. The dependency on $r_{S}$ explains why the $f_{n}^{C}$ shift to lower frequencies in Fig. 4c, in comparison to Fig. 4a.

As frequency decreases further from $f_{n}^{C}$, there occurs yet another transition frequency $f_{n}^{C, N F}$ where the slope becomes less steep. These edges indicate a mode's transition from the far field to the near field of the radiating surface $V$. Comparison of all plots in Fig. 4 suggests that the transition frequency $f_{n}^{C, \mathrm{NF}}$ is inversely proportional to $r_{B}$. A closer inspection suggests that $f_{n}^{C, N F} \approx \frac{n \pi c}{2 \pi \kappa r_{B}}$ holds for the transition frequencies, which are marked for $n=1 \ldots 3$ in Fig. 4d. It follows implicitly for the given orders $n=0 \ldots L-1$ that the smaller the value of $r_{B}$ the wider the frequency range where the corresponding modes demonstrate near-field effects.

From the comparison of Fig. 4a and Fig. 4c, it can be deduced that a larger array can theoretically control the sound field it radiates with less effort at low frequencies than a smaller array.

Figure 5 shows the ARF for $\kappa=\pi$, which represents the infinite half-space. The transition frequencies $f_{n}^{C}$ shift towards lower frequencies for the wider wedge angle, as can be expected. The discrepancy between the magnitude of two consecutive evanescent modes is only half of that which can be seen for the case of $\kappa=\frac{\pi}{2}$. This had to be expected since the order $\frac{n \pi}{\kappa}$ of the Hankel functions contributing to

\footnotetext{
${ }^{2}$ Note that all graphs given with an ordinate in $\mathrm{dB}$ are referred to unity.
}

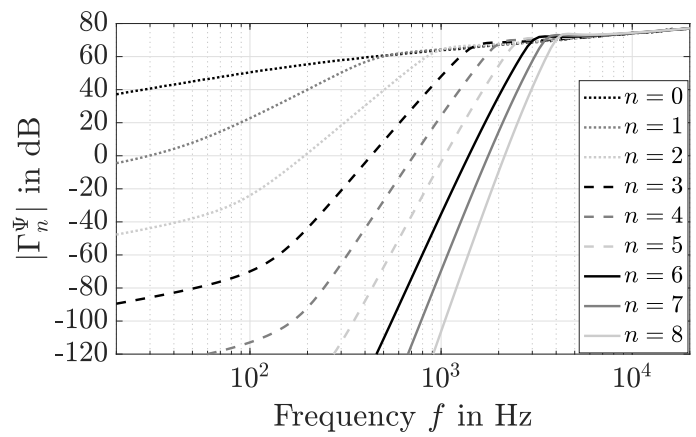

(a)

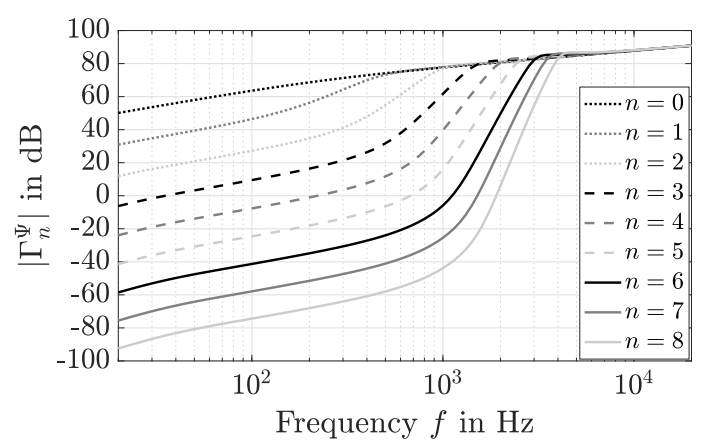

(b)

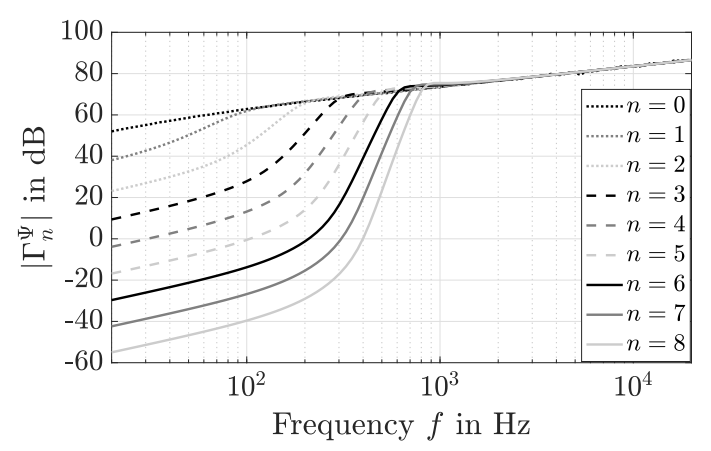

(c)

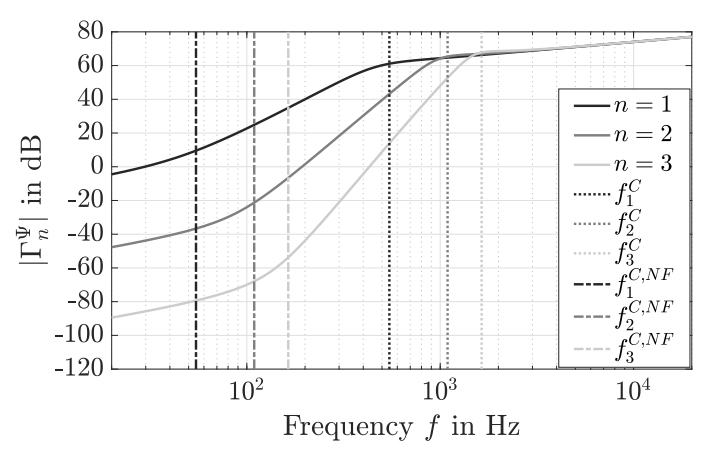

(d)

Figure 4: Magnitude of the $\operatorname{ARFs} \Gamma_{n}^{\Psi}$ with the parameters $\kappa=\frac{\pi}{2}$, $L=15, z_{S}=z_{B}=0 \mathrm{~m}$ and (a) $r_{B}=2 \mathrm{~m}, r_{S}=0.2 \mathrm{~m}$, (b) $r_{B}=0.5 \mathrm{~m}$, $r_{S}=0.2 \mathrm{~m}$, (c) $r_{B}=2 \mathrm{~m}, r_{S}=1 \mathrm{~m}$, and (d) $r_{B}=2 \mathrm{~m}, r_{S}=1 \mathrm{~m}$ with indicated transition frequencies.

$\Gamma_{n}$ effectively increases twice as fast for $\kappa=\frac{\pi}{2}$ than for $\kappa=\pi$ (see Eq. (3)).

The integral in Eq. (24) was calculated numerically using the procedure described in [34]. The bounds were 


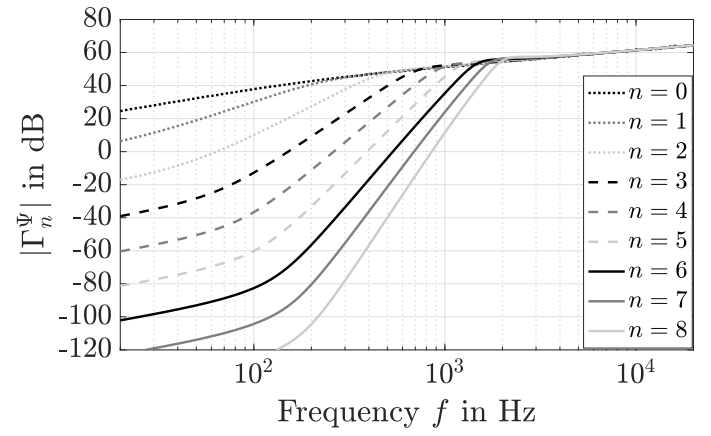

Figure 5: Magnitude of the ARFs, $\Gamma_{n}^{\Psi}$, with $\kappa=\pi, L=15, r_{B}=2 \mathrm{~m}$, $r_{S}=0.2 \mathrm{~m}, z_{S}=z_{B}=0 \mathrm{~m}$.

chosen as $-K_{z}$ and $K_{z}$, where

$$
K_{z}=\left\lceil\frac{Q k}{\Delta k_{z}}\right\rceil \Delta k_{z}
$$

with a step size $\Delta k_{z}=0.01$. The parameter $Q$ adjusts the range $k_{z}$ and is chosen so that the bounds include all components for $n=0$ that satisfy $20 \log _{10}\left(\left|\Gamma_{0}\left(k_{z}, r_{B}\right)\right|\right)>$ $-150 \mathrm{~dB}$. Note that $\lceil\cdot\rceil$ represents the ceiling function.

While numerical integration may be one way to calculate the ARFs with very good approximation, it is computationally expensive. The Stationary Phase Approximation (SPA) of the expression in Eq. (24) (see Appendix A) yields the far-field approximation for the ARF

$$
\Gamma_{n, \mathrm{FF}}^{\Psi}(R, \theta) \approx \frac{\rho_{0} c L}{\kappa \pi} \mathrm{e}^{-\mathrm{i} \frac{n \pi^{2}}{2 \kappa}} \frac{\mathrm{e}^{\mathrm{i} k R}}{R} \frac{V_{n}^{D}(k \cos \theta) \mathrm{e}^{-\mathrm{i} k z_{S} \cos \theta}}{\sin \theta H_{\frac{n \pi}{\kappa}}^{\prime}\left(k \sin \theta r_{S}\right)} .
$$

The SPA of $\Gamma_{n}^{\Psi}\left(r_{B}, z_{B}\right)$ provides a result with good accuracy when $r_{B}$ corresponds to the far field. Its expression has the advantage over Eq. (24) that it is given in analytic form and is thus fast to compute. It can be used alternatively in Eq. (25).

The difference between the ARFs and their far-field approximations when evaluated in the near field becomes clear when comparing the results of Fig. $4 \mathrm{a}$ and Fig. 6. At

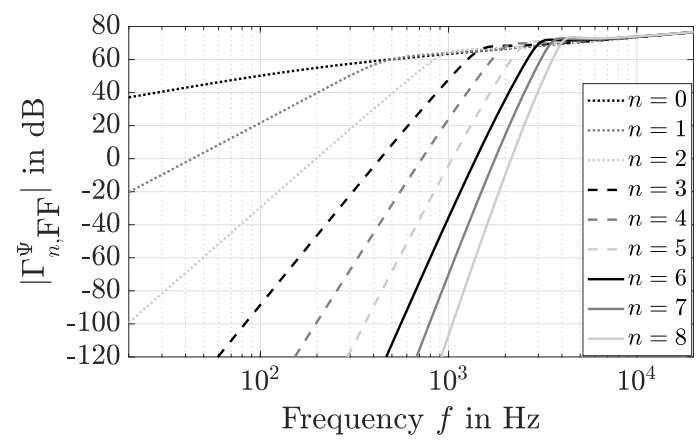

Figure 6: Magnitude of the far-field ARFs, $\Gamma_{n, \mathrm{FF}}^{\Psi}, \kappa=\frac{\pi}{2}, L=15$, $R=r_{B}=2 \mathrm{~m}, \theta=\frac{\pi}{2}, z_{S}=0 \mathrm{~m}$.

high frequencies there is hardly any difference, except for the small error of the numerical integration process. Below the edge frequencies $f_{n}^{C}$, the magnitudes of $\Gamma_{n, \mathrm{FF}}^{\Psi}$ decrease monotonically with frequency, unlike the values of $\Gamma_{n}^{\Psi}$ that change decay once more at $f_{n}^{C, N F}$. This difference and its relation to the transition frequencies $f_{n}^{C, N F}$ becomes clear when looking at the ratio of $\Gamma_{n}^{\Psi}$ and its SPA shown in Fig. 7. As with the magnitude, the phases of $\Gamma_{n}^{\Psi}$ and

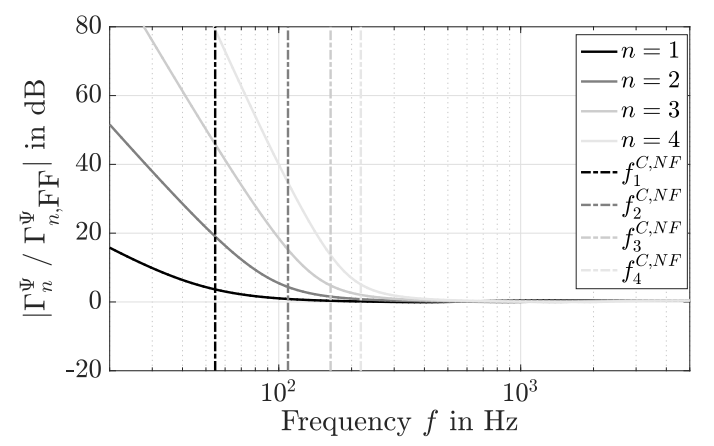

Figure 7: Magnitude of the ratio of $\Gamma_{n}^{\Psi}$ and $\Gamma_{n, \mathrm{FF}}^{\Psi}, \kappa=\frac{\pi}{2}, L=15$, $R=r_{B}=2 \mathrm{~m}, \theta=\frac{\pi}{2}, z_{S}=z_{B}=0 \mathrm{~m}$.

$\Gamma_{n, \mathrm{FF}}^{\Psi}$ also show a discrepancy below $f_{n}^{C}$. Using the SPA of the ARF thus has an impact on the array performance at low frequencies and for small $r_{B}$, as will be shown in Section 3.7.

Since the edge frequencies $f_{n}^{C}$ do not change between the ARF and their far-field approximations, choosing one or the other for the calculation of the driving functions has no influence on the array's spatial aliasing performance.

\subsection{Truncation of Higher Order Modes}

For a given frequency, the behaviour of both types of arc radial functions, $\Gamma_{n}^{\Psi}$ and $\Gamma_{n, \mathrm{FF}}^{\Psi}$, indicates that all modes above an order $n_{E}$ show evanescent behaviour in their decay with distance. This mode, and all subsequent modes, thus significantly lose their contribution to the overall sound field as they propagate. It can thus be assumed that there is an upper edge frequency where a truncation of the modelling order to $n=N$ has a negligible effect on the pressure $p\left(r_{B}, \phi, z_{B}\right)$. If then $N \leq L-1$, it follows from Eq. (22) that $A_{n, m}=\delta_{n-m}$, so that the truncation applied to the expression in Eq. (25) yields the approximation

$p\left(r_{B}, \phi, z_{B}\right) \approx \sum_{n=0}^{N} \Gamma_{n}^{\Psi}\left(r_{B}, z_{B}\right) Q_{n}(\omega) \Phi_{n}(\phi)=\sum_{n=0}^{N} P_{n} \Phi_{n}(\phi)$,

which itself can be described comprehensively by a set of coefficients $\left\{P_{n}\right\}_{n=0}^{N}$. Comparing factors between the two expressions in Eq. (27) and reorganising for $Q_{n}$ yields

$$
Q_{n}=\frac{1}{\Gamma_{n}^{\Psi}\left(r_{B}, z_{B}\right)} P_{n}
$$

This result is widely known and is typical a mode-matching expression [7, 14, 15, 18, 44]. 
Eq. (28) provides the driving function coefficients $Q_{n}$ as a function of the coefficients $P_{n}$ that define the desired pressure field on the arc $\Psi$. The values of $\Gamma_{n}^{\Psi}\left(r_{B}, z_{B}\right)$ describe how the different modes $Q_{n}$ of the loudspeaker driving function expansion in Eq. (17) radiate into the pressure field. It was shown in Section 3.5 that the arc radial functions can take values of very small magnitude at low frequencies. Their reciprocals in Eq. (28) then take very large values, effecting very high power demands for the individual loudspeakers [30]. In practice it is therefore necessary to apply regularisation to the inverse solution in Eq. (28) to protect the mechanical integrity of the loudspeakers [19] and, more generally, to ensure robustness and stability.

\subsection{Regularised Driving Functions}

Using a normalised Tikhonov regularisation, the inverse solution is reformulated to

$$
\tilde{Q}_{n}=\frac{\Gamma_{n}^{\Psi *}\left(r_{B}, z_{B}\right)}{\left|\Gamma_{n}^{\Psi}\left(r_{B}, z_{B}\right)\right|^{2}+\beta \sigma^{2}} P_{n}
$$

where $\sigma=\max _{n}\left|\Gamma_{n}^{\Psi}\left(r_{B}, z_{B}\right)\right|$. The parameter $\beta$ adjusts the tradeoff between low-frequency directivity and maximum displacement of the loudspeakers' diaphragms, where a limitation of the latter improves the robustness of the system [19]. Using Eq. (29) in Eq. (17) provides the regularised individual loudspeaker driving functions

$$
q_{l}=\sum_{n=0}^{L-1} \frac{\Gamma_{n}^{\Psi *}\left(r_{B}, z_{B}\right)}{\left|\Gamma_{n}^{\Psi}\left(r_{B}, z_{B}\right)\right|^{2}+\beta \sigma^{2}} P_{n} \Phi_{n}\left(\phi_{l}\right)
$$

for a desired pressure profile specified by the set $\left\{P_{n}\right\}_{n=0}^{N}$. The fact that $r_{B}$ remains a parameter makes for the main difference to the driving functions presented in [30], where the (arc) radial functions were derived from a plane wave scattering model through reciprocity and hence cannot be adjusted to a particular control radius. If $r_{B}$ is large enough to be in the far field for all frequencies of interest, then there is no difference between using either $\Gamma_{n}^{\Psi}$ or $\Gamma_{n, \mathrm{FF}}^{\Psi}$ for the calculation of $q_{l}$. The difference in performance of the driving functions when using the two types of ARFs is investigated in Section 6.

\section{Beamforming}

As with other geometric array arrangements, beamforming with wedge arrays aims to control the radiated pressure field in such a way that the pressure magnitude on the control arc $\Psi$ resembles a designated beam pattern.

In this work, two different types of beam patterns are considered. The first approximates a Dirac pulse in the $\phi$-domain, and the second corresponds to a Rectangular pulse of variable width $D$. Both are approximated using finite expansions of the form

$$
f(\phi)=\sum_{n=0}^{N} F_{n} \Phi_{n}(\phi),
$$

so that the two beam patterns differ with respect to their coefficients $F_{n}$. These can be calculated using

$$
F_{n}=\mathcal{W}[f(\phi) \delta(z)]
$$

To ensure a unit magnitude in the steering direction $\phi_{B}$, it is necessary to normalise these coefficients by the factor $\sum_{m=0}^{N} F_{m} \Phi_{m}\left(\phi_{B}\right)$. The exact expressions for the coefficients used in this work are presented below.

\subsection{Beam Pattern I}

The coefficients $F_{n}^{I}$ of the beam pattern approximating a Dirac pulse are given by

$$
F_{n}^{I}=\frac{\Phi_{n}\left(\phi_{B}\right)}{\sum_{m=0}^{N} \Phi_{m}\left(\phi_{B}\right)^{2}} .
$$

Some examples of beam pattern I with $N=10$ are shown in Figure 8 for values of $\phi_{B}$.

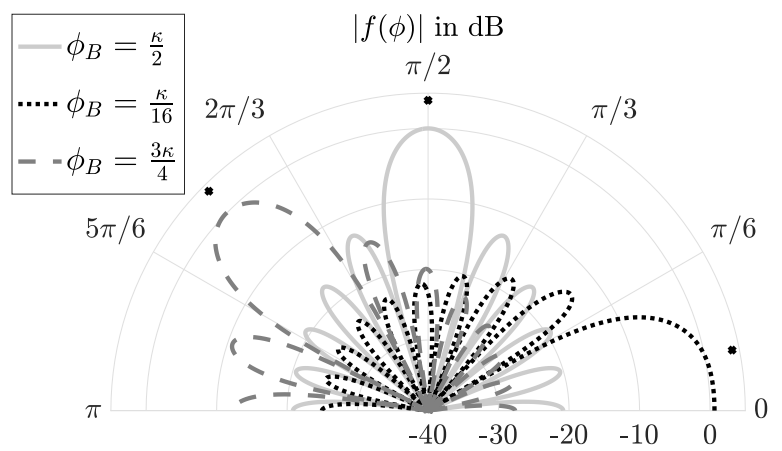

Figure 8: Examples for Beam Pattern I with different steering angles $\phi_{B}, \kappa=\pi, N=10$.

\subsection{Beam Pattern II}

The coefficients $F_{n}^{I I}$ of the beam pattern corresponding to a rectangular pulse of width $D$ are given by

$$
F_{n}^{I I}=\frac{D \operatorname{sinc}\left(\frac{n \pi}{\kappa} \frac{D}{2}\right) \Phi_{n}\left(\phi_{B}\right)}{\sum_{m=0}^{N} D \operatorname{sinc}\left(\frac{m \pi}{\kappa} \frac{D}{2}\right) \Phi_{m}\left(\phi_{B}\right)^{2}} .
$$

Beam pattern II is shown in Figure 9 for different steering angles. Unlike pattern I, pattern II enables control over the width of the main lobe.

Comparing the results in Figure 8 and Figure 9 shows that by allowing for an increased beam width with pattern II, the side lobes' magnitude decreases significantly w.r.t. that of the side lobes of pattern I. There appears to be a trade-off between the width of the main lobe and its difference in magnitude compared to the side lobes (ie. the acoustic contrast). It can also be observed that the number of side lobes reduces slightly for Type II beams.

With a fully developed theory for sound field control with wedge arrays at hand, their performance shall be initially evaluated on the basis of simulations. 


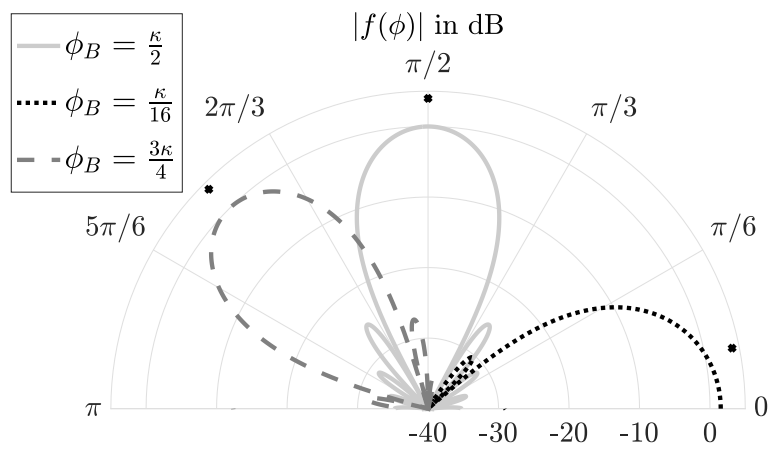

Figure 9: Examples for Beam Pattern II with different steering angles $\phi_{B}$ and $D=0.2 \pi, \kappa=\pi, N=10$.

\section{Simulations}

This section shows simulation results for the beamforming performance of wedge arrays with two wedge angles, a Quadrant Cylindrical Array (QCA), with $\kappa_{1}=\frac{\pi}{2}$, and a Hemi-Cylindrical Array (HCA), with $\kappa_{2}=\pi$. The former corresponds to an array that is located in the corner of a room with rigid walls while the latter can be an array located on any large rigid wall. Note that all beam patterns presented in the remainder of this work include a black line that indicates the points where the main lobe has dropped by $6 \mathrm{~dB}$ from its maximum magnitude.

The radiated beam patterns were calculated on the basis of the sound field synthesis model in (2) for the plane $z=z_{B}=z_{S}$, where the infinite sum over $n$ was truncated to $n=30$ for the QCA and to $n=60$ for the HCA. The speed of sound was assumed to be $c=343 \frac{\mathrm{m}}{\mathrm{s}}$ and the specific density of air $\rho_{0}=1.2041 \frac{\mathrm{kg}}{\mathrm{m}^{3}}$. The velocity spectrum was calculated using Eq. (13), where different types of source models were used for the QCA and the HCA. The loudspeaker driving functions were obtained from Eq. (30), using the ARF in Eq. (24), unless stated otherwise. The simulations cover the two beam pattern types given in Eq. (33) and Eq. (34), and different control radii. All results are given within the frequency band from $100 \mathrm{~Hz}$ up to $10 \mathrm{kHz}$.

\subsection{Case Study: Quadrant Cylindrical Array with $L=15$}

For the QCA with $L=15$, all loudspeakers were modelled as point sources, i.e. $V_{n}^{D}\left(k_{z}\right)=1$, with the velocity spectrum $V_{n}\left(k_{z}\right)$ as defined in Eq. (13). Figure 10 shows the normalised reproduced beam pattern of a QCA with $r_{S}=0.2 \mathrm{~m}$ for the target patterns Type I and Type II, depicted on the right side of the figure. The lobe structures resemble that of the corresponding targets in the array's optimal frequency range between 5 and $8 \mathrm{kHz}$. Below that, as frequency decreases, the beam broadens while the number of side lobes drops. The side lobes remain distinct until directivity decreases so much that only the main lobe is left. This is founded in the required regularisation of the inverse solution in Eq. (30) with $\beta=10^{-4}$ (see Section 3.7) [19]. The described effect is inherent to all types of wedge

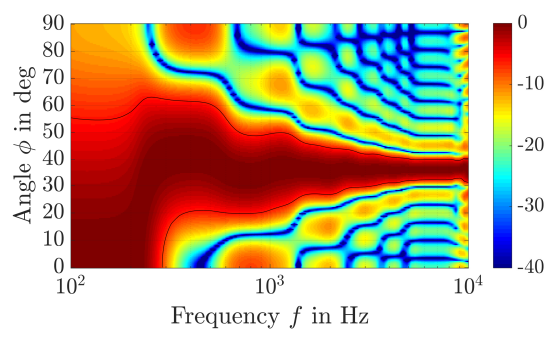

(a)

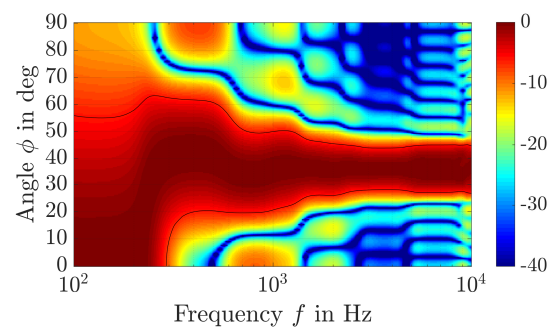

(b)
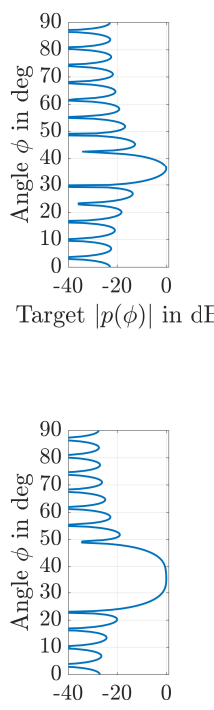

Target $|p(\phi)|$ in $\mathrm{dB}$
Figure 10: (Colour online) Simulated beam patterns for a QCA with $L=15, N=14, \beta=10^{-4}, \phi_{B}=36^{\circ}, r_{B}=2 \mathrm{~m}$ and $r_{S}=0.2 \mathrm{~m}$, (a) Beam Pattern Type I, (b) Beam Pattern Type II, $D=0.2 \frac{\pi}{2}$.

arrays and will not be explicitly mentioned when discussing the simulation results presented below.

Figure 11 shows the beam pattern when the far-field arc radial functions $\Gamma_{n, \mathrm{FF}}^{\Psi}$ were used to calculate the driving functions. The depicted beam patterns best approximate

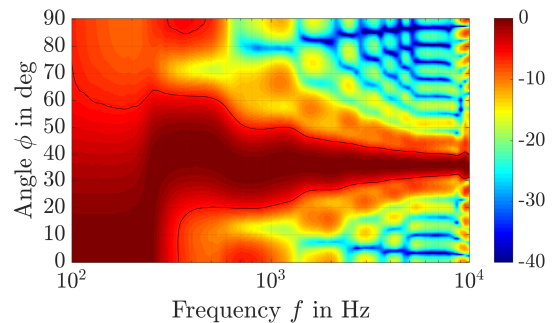

(a)

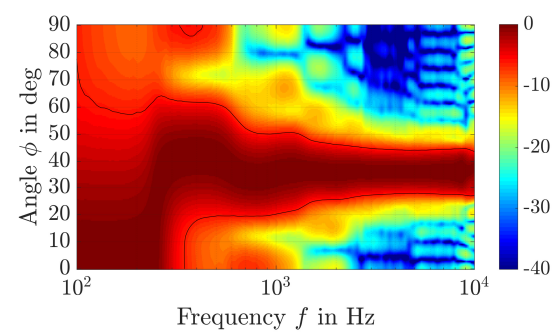

(b)
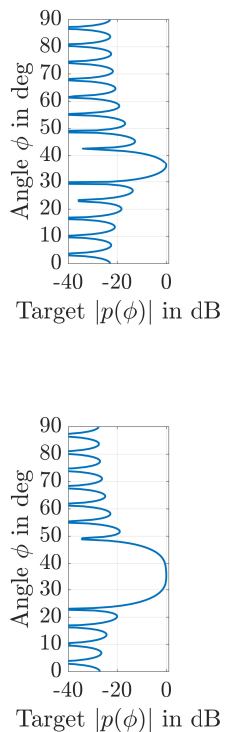

Figure 11: (Colour online) Simulated beam patterns based on farfield ARFs for a QCA with $L=15, N=14, \beta=10^{-4}, \phi_{B}=36^{\circ}$, $r_{B}=2 \mathrm{~m}$ and $r_{S}=0.2 \mathrm{~m}$, (a) Beam Pattern Type I, (b) Beam Pattern Type II, $D=0.2 \frac{\pi}{2}$.

the target within the aforementioned optimal frequency range, while, for most part of the displayed frequency range, the individual lobes are smeared and not as distinct 
as the target. This effect appears to increase towards low frequencies and can be traced back to the lack of control that the far-field ARFs provide at $r_{B}=2 \mathrm{~m}$. This result suggests that the sound field still shows a near-field dominated behaviour for $r_{B}=2 \mathrm{~m}$ and therefore requires the use of the ARF in Eq. (24) to calculate the driving functions for the best control of the beam pattern.

Spatial aliasing effects are visible in both Fig. 11 and Fig. 10 for frequencies higher than $8 \mathrm{kHz}$.

Figure 12 shows the beam pattern (Type I) radiated from a QCA with the same parameters as before apart from $r_{S}=1 \mathrm{~m}$. Since the number of loudspeakers is the
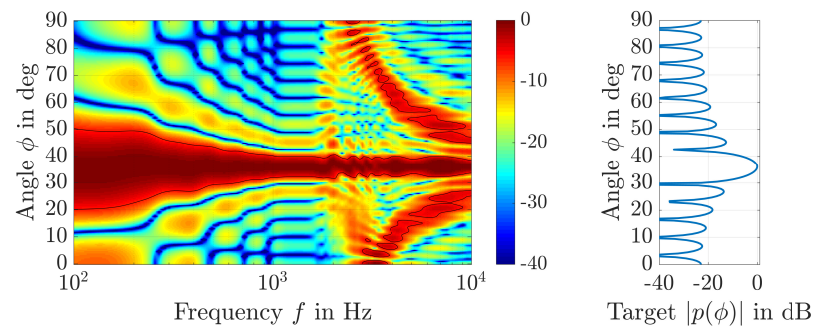

Figure 12: (Colour online) Simulated beam pattern for a QCA with $L=15, N=14, \beta=10^{-4}, \phi_{B}=36^{\circ}, r_{B}=2 \mathrm{~m}$ and $r_{S}=1 \mathrm{~m}$.

same, the effects of spatial aliasing are already visible at much lower frequencies. However, the directivity at low frequencies has improved significantly, as it was predicted for arrays with larger radius $r_{S}$ in Section 3.5.

All simulations with the QCA show a certain variability of the angle at the main lobes' centre, particularly at low frequencies, where it deviates from the desired steering angle $\phi_{B}$.

In principle, the observations described in this subsection for the QCA are transferable to any other wedge array. The next subsection shows the simulation of the performance of the HCA.

\subsection{Case Study: Hemi Cylindrical Array with $L=15$}

This case study provides reference results for the experimental data presented in Section 6. The prototype, which was previously presented in [30] and [34], is depicted in Figure 13. It consists of $L=15$ Tang Band W1-1070SG full-range drivers positioned at the angles

$$
\phi_{l}=\frac{\pi}{L+1} l, l=1 \ldots L
$$

and at the corresponding $z$-axis values

$$
z_{l}=z_{B}+\left[-z_{S},+z_{S},-z_{S}, \ldots,-z_{S}\right]
$$

where $z_{S}=0.0275 \mathrm{~m}$.

Unlike with the QCA, each loudspeaker in the HCA is modelled as a rectangular piston, using the coefficients $V_{n}^{D}\left(k_{z}\right)$ as defined in Eq. (12) with $B=0.031 \mathrm{~m}$ and $\Theta \approx 0.22$. Even though this seems an unusual choice to model a circular diaphragm, it must be said that the

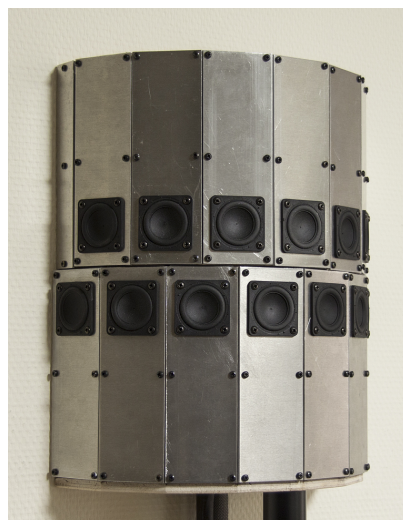

Figure 13: (Colour online) Prototype of a HCA with $r_{S}=14.2 \mathrm{~cm}$ and $L=15$ Tang Band $W 1-1070 S G$ full-range drivers.

unavoidable order limitation effectively 'rounds the corners' of the modelled pistons, thus reducing the discrepancy.

The interleaved design aims to keep the array compact while increasing the number of drivers to gain a higher degree of spatial control. With this choice of $\phi_{l}$ and $z_{l}$, however, the evaluation of the sum in Eq. (21) does not yield the result in Eq. (22) for the interleaved arrangement. Instead, one obtains a more complex relationship for the elements of the orthogonality matrix $A_{n, m}$, which is shown in Figure 14a. Sampling the modes at the loudspeaker

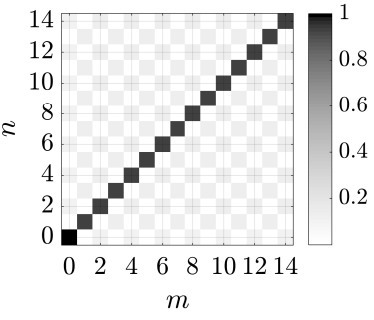

(a)

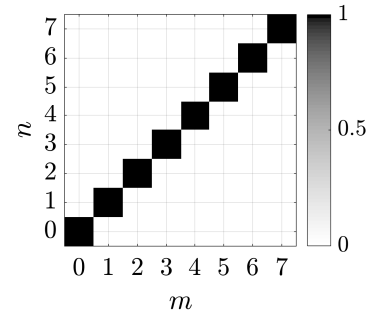

(b)
Figure 14: Visualisation of the magnitude of the orthogonality matrix $A_{n, m}$ for (a) the full prototype with $L=15$ and (b) the bottom layer only with $L=8$.

positions of the interleaved design no longer preserves the orthogonality of modes. That means when attempting to control the $m$ th order, the system actually excites the $m$ th order but leaks energy into other orders as well. This is undesirable for a mode-matching approach, which assumes that only the modes it aims to control receive energy from the system. The prototype design is not orthogonal because it is missing 'half' a speaker at each end of the aperture, i.e. $\phi=0, \pi$. If one uses only the bottom layer of the prototype (8 loudspeakers), the coefficients $A_{n, m}$ describe a perfect diagonal with all off-diagonal elements equal to zero, as depicted in Figure 14b. Despite the orthogonality flaw in the interleaved design, the loudspeaker driving functions were still calculated with (30).

The performance of the full prototype for the target 
patterns Type I and II is shown in Figure 15. The maximum
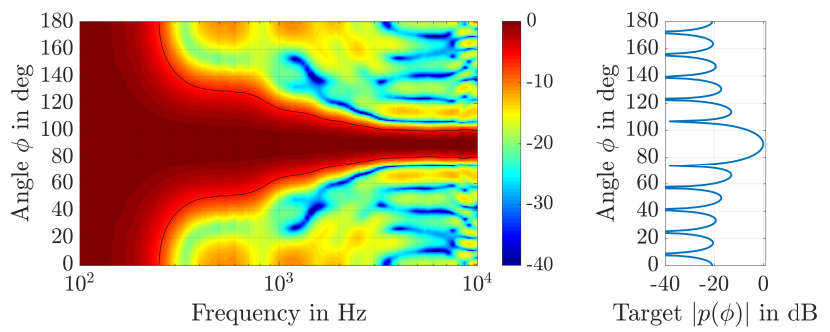

(a)

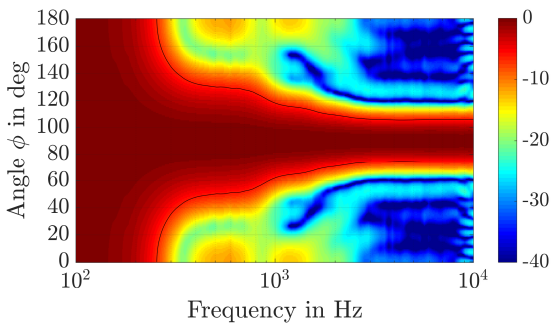

(b)

Figure 15: (Colour online) Simulated beam patterns for an HCA with $L=15, N=10, \beta=0.1, \phi_{B}=\frac{\pi}{2}$ and $r_{B}=2 \mathrm{~m}$, (a) Beam Pattern Type I, (b) Beam Pattern Type II, $D=0.2 \pi$.

control order $N=L-1$ was reduced to 10 in order to push the lower aliasing edge frequency higher [19]. The result for the target Type I shows the highest directivity, while the array's performance with target Type II yields a very high side lobe suppression above $3 \mathrm{kHz}$. As it was observed for the QCA, the regularisation causes a reduction of directivity as frequency decreases. For both targets, the simulation shows a relatively stable beam width in the frequency range from 3 to $8 \mathrm{kHz}$; Spatial aliasing begins breaking up the beam pattern at frequencies beyond the latter frequency.

Figure 16 shows the reproduced target beam patterns for the prototype's bottom layer only. When compared to Fig. 15a, as it can be expected, the frequency above which spatial aliasing occurs is much higher for interleaved configuration than for the bottom layer array. Aliasing effects start to show above $3 \mathrm{kHz}$ for target Type I and only slightly further up for Type II. This effect is the result of the reduced magnitude of the coefficients $F_{n}$ for higher values of $n$ in the case of the Type II pattern.

It can also be seen that the full prototype does not reproduce the distinct target pattern as the bottom layer alone. Instead the side lobes in Figure 15 are smeared, independently of the type of target. This was observed before for the QCA, however, it was found to be caused by the imperfection of the far-field ARF. In this case it appears that the lack of orthogonality inherent to the loudspeaker positions is responsible for this. However, even though the side lobes can no longer be clearly distinguished, their magnitude is lower than with the bottom layer alone, showing that there is still a benefit from the interleaved layout. Furthermore, as it was already predicted in Sec-

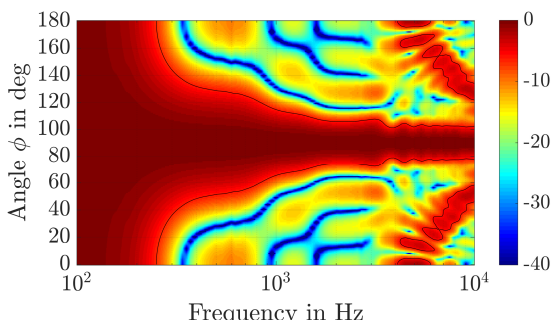

(a)

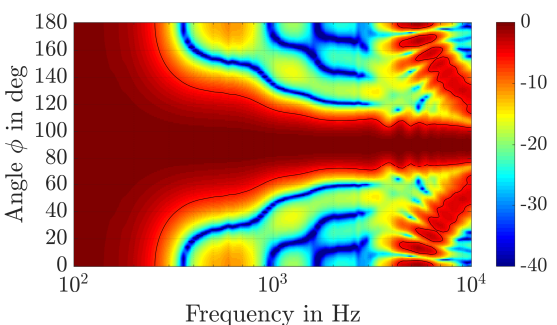

(b)
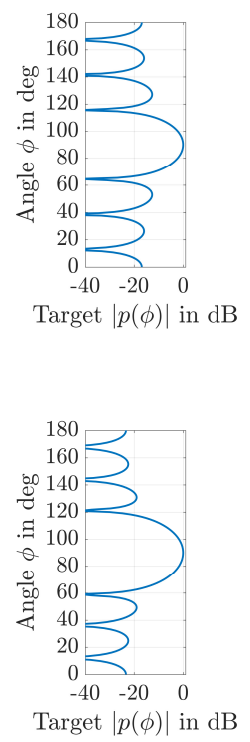

Target $|p(\phi)|$ in $\mathrm{dB}$
Figure 16: (Colour online) Simulated beam patterns for an HCA with $L=8, N=7, \beta=0.1, \phi_{B}=\frac{\pi}{2}$ and $r_{B}=2 \mathrm{~m}$, (a) Beam Pattern Type I, (b) Beam Pattern Type II, $D=0.2 \pi$.

tion 4, target Type II is shown to reduce the magnitude of the side lobes significantly over those achieved with target Type I.

It was found that decreasing the regularisation factor $\beta$ does not improve the beam width at low frequencies for the interleaved configuration, yet it does so for the bottom layer. Therefore, in theory, the bottom layer by itself can yield a narrower beam at low frequencies than the interleaved configuration. This suggests that effects due to lack of orthogonality can also be mitigated by relatively strong regularisation, at the cost of reduced directivity at low frequencies. Alternatively, the artefacts occurring with the mode-matching approach may be more controllable when choosing a pressure-matching approach instead [18].

The next two simulations consider only target Type I. The performance for the interleaved array with different steering angles $\phi_{B}$ in Figure 17 shows that the integrity of the main lobe remains relatively well preserved at frequencies beyond $3 \mathrm{kHz}$. The side lobes for the relatively extreme steering angle of $\phi_{B}=\frac{5 \pi}{6}$ rise up to just under $6 \mathrm{~dB}$ below the magnitude of the main lobe. It was found that this effect can be mitigated with a beam pattern Type II. At lower frequencies however, it appears that the main lobe no longer stays centred around the steering angle. This was already observed during the case study of the QCA and appears to occur only for steering angles $\phi_{B} \neq \frac{\kappa}{2}$. A possible cause of this phenomenon is the combination of asymmetry and the break up of the lobe structure as a result of regularisation. 


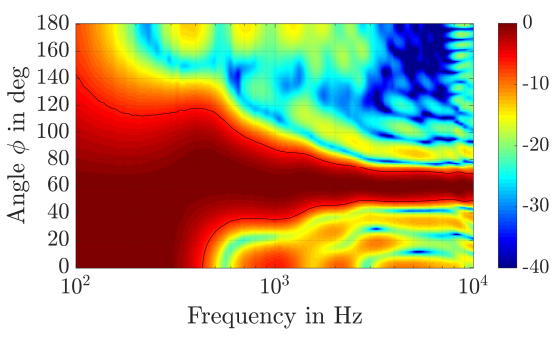

(a)

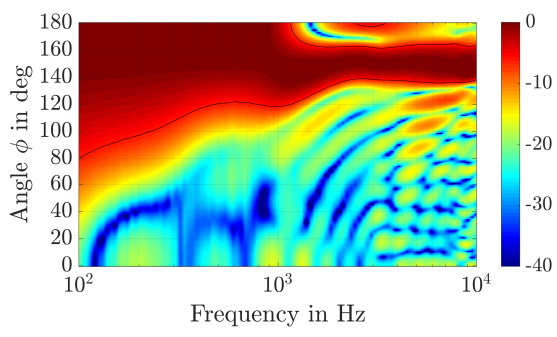

(b)

Figure 17: (Colour online) Theoretically achievable beam patterns for an array with $L=15$ speakers, with $N=10, \beta=0.1, r_{B}=2 \mathrm{~m}$, and (a) $\phi_{B}=\frac{\pi}{3}$ and (b) $\phi_{B}=\frac{5 \pi}{6}$.

\section{Measurements}

\subsection{Setup}

Measurements to confirm the model for sound field control with wedge arrays were conducted for the case of hemi-cylindrical loudspeaker arrays. For this purpose, the prototype described in the previous section was placed on a large planar baffle inside an anechoic chamber. Absorbing material (glass wool wedges) was placed at the edges of the planar baffle to reduce reflections. The prototype was extended with wooden hemi-cylinders to better match the assumption of an infinite hemi-cylinder.

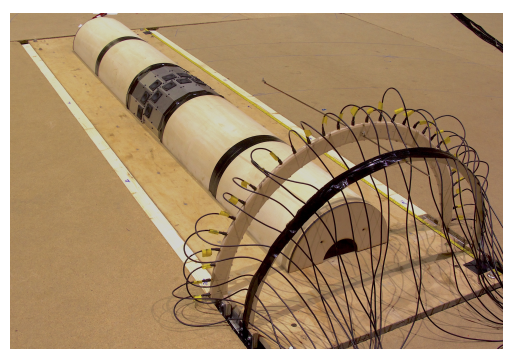

Figure 18: (Colour online) The measurement setup with a measurement array consisting of 30 G.R.A.S. 4OPL array microphones fitted on an arch over the $\mathrm{HCA}$ with $30 \mathrm{~cm}$ radius.

The measurement arc with 30 G.R.A.S. 40PL array microphones is shown in Figure 18.

This measurement arc was translated along the vertical axis of the wedge array between measurements to acquire holograms of the radiated sound field on a hemi-cylindrical surface around the array at the radius $r_{H}=0.3 \mathrm{~m}$. From this hologram data, it is possible to reconstruct the pressure field at different points in the propagation space by means of acoustical holography. This process is fully described for the wedge geometry in [34], along with a more detailed description of the measurement rig. The speed of sound and the specific density of air were chosen to match the in situ conditions, $c=342.102 \frac{\mathrm{m}}{\mathrm{s}}$ and $\rho_{0}=1.2130 \frac{\mathrm{kg}}{\mathrm{m}^{3}}$.

Unless mentioned otherwise, all measurement results have been reconstructed at the control radius $r_{C}=2 \mathrm{~m}$ and the height $z_{B}$ (i.e. the centre between the two array layers), where the holographic reconstruction of the pressure is denoted as $\hat{p}\left(r_{C}, \phi, z_{B}\right)$. The driving functions were calculated from (30). Unless stated otherwise, the steering angle was $\phi_{B}=60^{\circ}$ and the far-field $\operatorname{ARF} \Gamma_{n, \mathrm{FF}}^{\Psi}$ were used to calculate the driving functions.

\subsection{Normalised Mean Squared Measurement Error}

In order to assess the quality of the measurement data it was necessary to implement a measure that quantifies the error of the measured data $\hat{p}\left(r_{C}, \phi_{w}, z_{B}\right)$ with reference to the prediction $p\left(r_{C}, \phi_{w}, z_{B}\right)$ from the model. This work defines the normalised mean squared measurement error as

$$
\bar{e}(\omega)=\frac{\sum_{w=1}^{W}\left|\hat{p}\left(r_{C}, \phi_{w}, z_{B}\right)-p\left(r_{C}, \phi_{w}, z_{B}\right)\right|^{2}}{\sum_{w=1}^{W}\left|p\left(r_{C}, \phi_{w}, z_{B}\right)\right|^{2}},
$$

where $\phi_{w}$ denotes all discrete angles where the sound field was reconstructed from hologram data. Note that this definition of $\bar{e}(\omega)$ also takes the phase error into account.

All subplots shown in Figure 19 present the corresponding mean measurement error along with the reconstruction data.

\subsection{Results for Different HCA Beamforming Filters}

The results of the HCA performance measurements are shown in Figure 19 for a selection of different parameters, along with the measurement error. Unless explicitly stated otherwise, all filters were calculated on the basis of the far-field ARF, with $N=10$ on the basis of beam pattern Type I.

Figures 19a, 19b and 19c show the performance of the HCA for different steering angles. Figures 19a and 19e show the performance of the HCA for Beam Pattern Type I and Type II, respectively, at $\phi_{B}=60^{\circ}$. Figures 19a, 19f, $19 \mathrm{~g}$ and $19 \mathrm{~h}$ show the performance of the HCA for Beam Pattern Type I at $\phi_{B}=60^{\circ}$ for four different filter settings, namely:

1. FF ARF \& $r_{C}=2 \mathrm{~m}$,

2. NF ARF with $r_{B}=2 \mathrm{~m} \& r_{C}=2 \mathrm{~m}$,

3. $\mathrm{FF} \mathrm{ARF} \& r_{C}=0.25 \mathrm{~m}$, and

4. NF ARF with $r_{B}=0.25 \mathrm{~m} \& r_{C}=0.25 \mathrm{~m}$.

The mean measurement error remains below $20 \mathrm{~dB}$ for the largest part of the frequency spectrum, which supports the model and the measurement technique. 

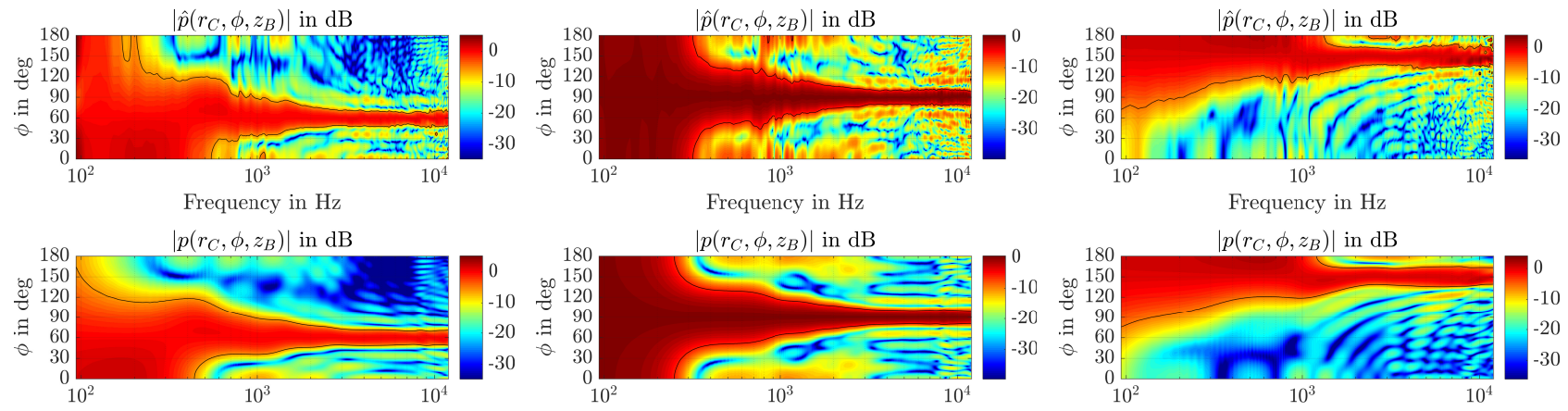

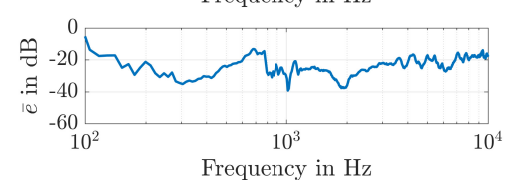

(a)

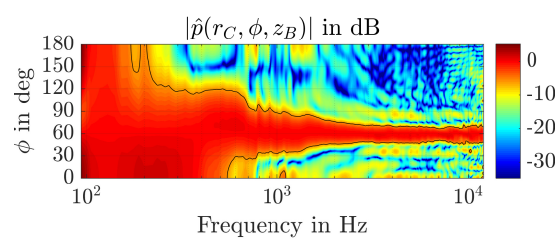

$\left|p\left(r_{C}, \phi, z_{B}\right)\right|$ in $\mathrm{dB}$
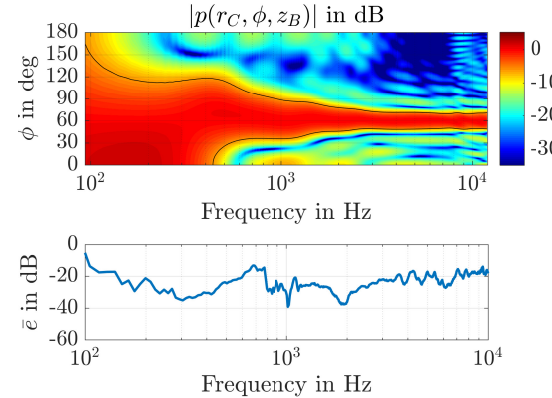

(d)

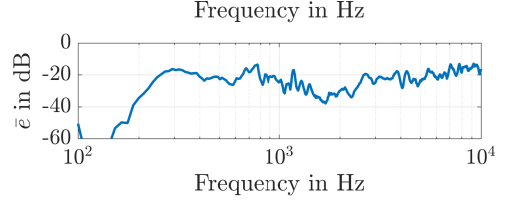

(b)

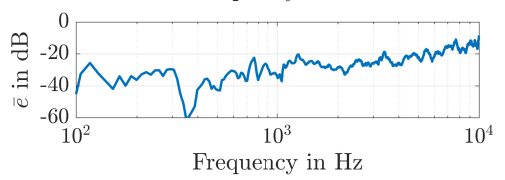

(c)

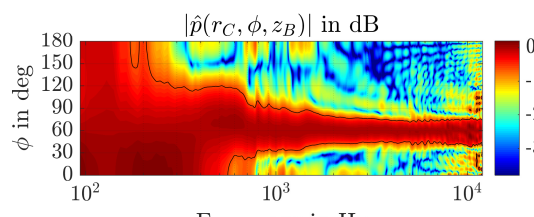

Frequency in $\mathrm{Hz}$

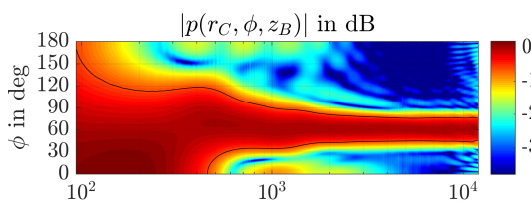

Frequency in $\mathrm{Hz}$

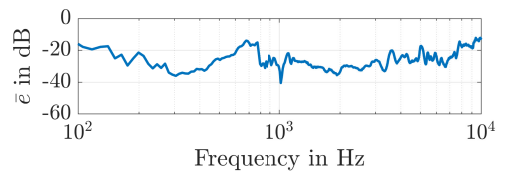

(e)

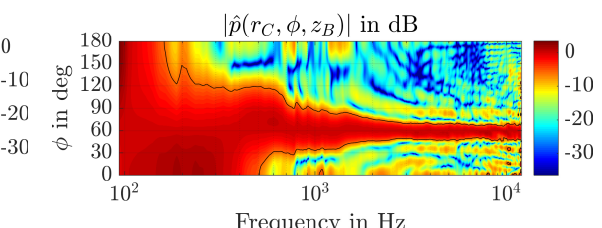

Frequency in $\mathrm{Hz}$

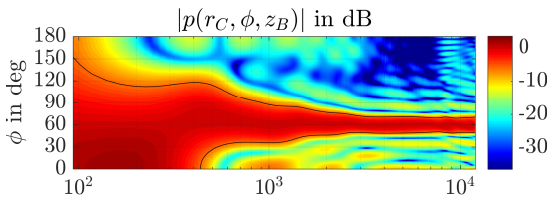

Frequency in $\mathrm{Hz}$

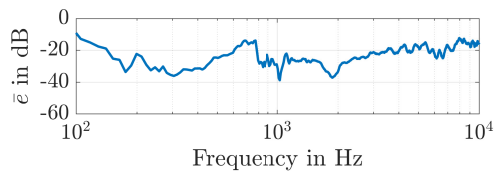

(f)
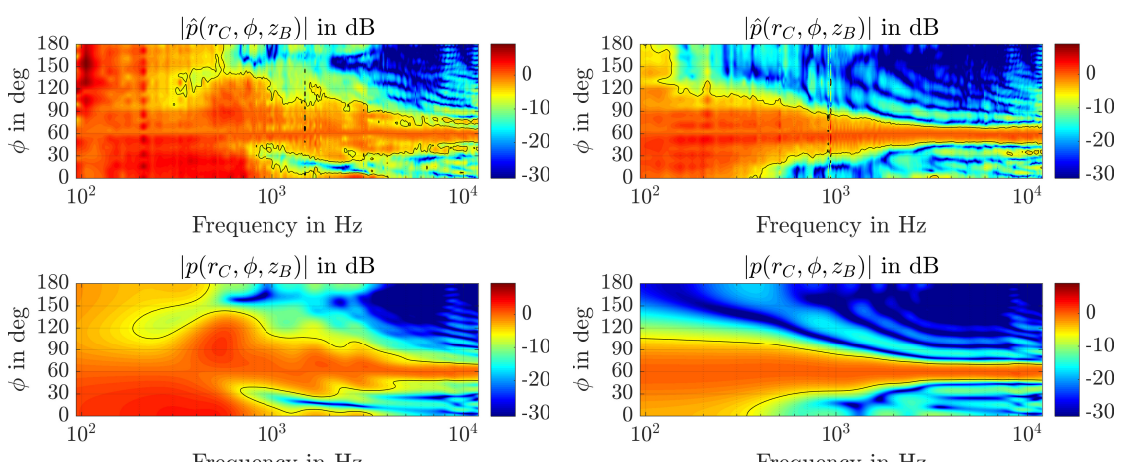

$\left|p\left(r_{C}, \phi, z_{B}\right)\right|$ in $\mathrm{dB}$

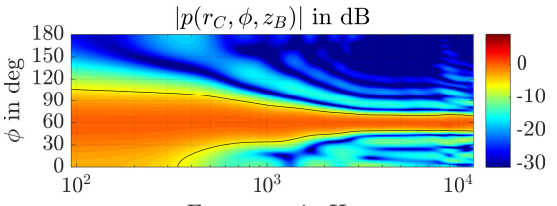

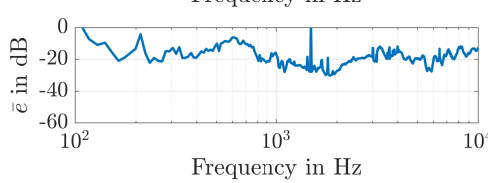

(g)

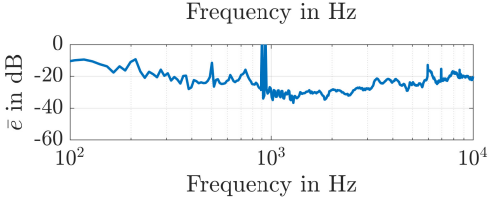

(h)

Figure 19: (Colour online) Normalised beam patterns (top) $\hat{p}\left(\phi, r_{C}, z_{B}\right)$ from holography, (centre) $p\left(\phi, r_{C}, z_{B}\right)$ from simulation, and (bottom) the normalised mean squared error $\bar{e}(\omega)$ for different steering angles $\phi_{B}$, beam patterns and arc radial filters, where (a) $\phi_{B}=60^{\circ},(\mathrm{b}) \phi_{B}=90^{\circ}$, (c) $\phi_{B}=150^{\circ}$, (d) BP I, (e) BP II, (f) NFs at $r_{C}=2 \mathrm{~m}$, (g) FF at $r_{C}=0.25 \mathrm{~m}$, (h) NF at $r_{C}=0.25 \mathrm{~m}$. 


\subsection{Discussion}

The reconstruction from the measurement and the theoretical result show very good similarity, with all prominent features (i.e. side lobes, change of beam width, etc.) in relatively good agreement. The measurement mismatches are assumed to be due to positioning errors of the microphones, imperfections in the acoustic setup due to reflections and finite structure and measurement aperture, and low SNR at low frequencies due to relatively small HCA output.

Of particular interest are probably those results shown in Figs. 19f, 19g and 19h since they confirm a good performance of the far-field ARF for reproduction radii of at least $r_{C}=2 \mathrm{~m}$. At this radius, no performance difference can be observed between the ARF and their far-field approximations. However, it can also be seen from the results in Figures $19 \mathrm{~g}$ and $19 \mathrm{~h}$ that, at small distances, filters based on the numerically calculated ARFs provide the desired performance in the near field of the HCA, while far-field ARF-based filters fail to achieve the performance target.

The influence of the beam pattern type appears to be relatively small, yet, as it was predicted, the Type II beam pattern yields lower side lobes compared to the Type I beam pattern.

The results confirm the findings from the theoretical model, and hence indicate that the developed model for wedge loudspeaker arrays is valid and provides good results.

\section{Conclusions}

An analytical model for the sound field of wedge loudspeaker arrays was developed. Unlike with line arrays, circular/cylindrical arrays and spherical arrays, the acoustic analytical model for wedge arrays requires less simplifying assumptions to be made for the acoustic environment of the array. Reflections from the adjacent walls in which the wedge array is mounted are already accounted for by a set of boundary conditions, so that their reflections are utilised in the sound field control process. Of course, as with other arrays, subsequent reflections from other walls would still occur, but the field distortion introduced by them may not have a perceivable effect on the beam pattern, if they are sufficiently far away.

The forward model maps the vibration profile of a loudspeaker array arranged in the shape of an arc on a curved baffle to the sound pressure in the propagation space. The radiation mechanism is described in the modal domain. Solving the inverse problem for a uniform loudspeaker distribution yields an expression for the control filters used to drive the array and control the radiated beam pattern. From the inverse solution, the aliasing behaviour of the system is predicted.

To study the system's control performance at different radii and frequencies, the concept of Arc Radial Functions (ARFs) is introduced. These were calculated numerically, both in their general form and using a far-field approximation.
Based on the developed model, the sound fields for a QCA and an initial prototype of an HCA were simulated for different parameters and control filters to analyse the performance of wedge arrays. In order to confirm the data obtained from simulation, a series of measurements was conducted in a controlled environment. Using a special measurement rig, sound field holograms were acquired that enable acoustic holography to reconstruct the sound field at any given distance away from the prototype. The results obtained from this data confirm the simulation results and validate the developed model.

It was shown that for far-field control applications, the far-field filters yield results sufficiently similar to those obtained from the general filters. For near-field applications, however, it was shown that only the more accurate general filters provide adequate sound field control, as expected. The far-field filters presented in this work are effectively the same as those derived in [30], and the corresponding findings therefore apply to either of them.

Future work will extend the model for the loudspeaker driving functions to allow for $3 \mathrm{D}$ sound field control and to derive driving functions and beamforming methods for loudspeaker arrays in finite wedges, for which the acoustic model was presented by the authors in [34].

\section{Acknowledgements}

This work has been partially funded by the Engineering and Physical Science Research Council, the Royal Academy of Engineering and HUAWEI. E.G. Williams is supported by the US Office of Naval Research. The authors acknowledge the use of the IRIDIS High Performance Computing Facility, and associated support services at the University of Southampton, in the completion of this work.

\section{Appendix A. Stationary Phase Approximation of the Arc Radial Functions}

In [21], Williams presented a generic formula for the SPA of integrals with the form

$$
I(R)=\int_{-\infty}^{\infty} f\left(k_{z}\right) \mathrm{e}^{\mathrm{i} R g\left(k_{z}\right)} \mathrm{d} k_{z}
$$

that is given by

$$
I(R) \approx f\left(k_{z 0}\right) \mathrm{e}^{\mathrm{i} R g\left(k_{z 0}\right)} \mathrm{e}^{-\mathrm{i} \frac{\pi}{4}} \sqrt{\frac{2 \pi}{R\left|g^{\prime \prime}\left(k_{z 0}\right)\right|}}, g^{\prime \prime}\left(k_{z 0}\right)<0
$$

where $k_{z 0}$ denotes the stationary phase point that satisfies

$$
\left.\frac{\mathrm{d} g\left(k_{z}\right)}{\mathrm{d} k_{z}}\right|_{k_{z}=k_{z 0}}=0
$$

and $g^{\prime \prime}\left(k_{z}\right)$ is the second derivative of $g$ with respect to $k_{z}$. If $r \rightarrow \infty$, then the integral in Eq. (24) can be made of 
the form in Eq. (A.1) by replacing the Hankel function in $\Gamma_{n}\left(k_{z}, r\right)$ by its large argument asymptote [21]

$$
H_{n}(x) \sim \sqrt{\frac{2}{\pi x}} \mathrm{e}^{\mathrm{i}(x-n \pi / 2-\pi / 4)}
$$

and changing to spherical coordinates $r_{B}=R \sin \theta$ and $z_{B}=R \cos \theta$, yielding

$$
\begin{aligned}
& \Gamma_{n, \mathrm{FF}}^{\Psi}(R, \theta) \approx \\
& \frac{\mathrm{i} \rho_{0} c k L \mathrm{e}^{-\mathrm{i}\left(\frac{n \pi^{2}}{2 \kappa}+\frac{\pi}{4}\right)}}{\kappa \pi^{\frac{3}{2}} \sqrt{2 R \sin \theta}} \int_{-\infty}^{\infty} \underbrace{\frac{V_{n}^{D}\left(k_{z}\right) \mathrm{e}^{-\mathrm{i} k_{z} z_{S}}}{k_{r}^{\frac{3}{2}} H_{\frac{n \pi}{\kappa}}^{\prime}\left(k_{r} r_{S}\right)}}_{f\left(k_{z}\right)} \mathrm{e}^{\mathrm{i} R g\left(k_{z}\right)} \mathrm{d} k_{z}
\end{aligned}
$$

where

$$
g\left(k_{z}\right)=\sqrt{k^{2}-k_{z}^{2}} \sin \theta+k_{z} \cos \theta .
$$

Evaluating the integral in Eq. (A.5) using Eq. (A.2) yields the result of the SPA as the analytic expression for the far-field approximation of the ARFs

$$
\Gamma_{n, \mathrm{FF}}^{\Psi}(R, \theta) \approx \frac{\rho_{0} c L}{\kappa \pi} \mathrm{e}^{-\mathrm{i} \frac{n \pi^{2}}{2 \kappa}} \frac{\mathrm{e}^{\mathrm{i} k R}}{R} \frac{V_{n}^{D}(k \cos \theta) \mathrm{e}^{-\mathrm{i} k z_{S} \cos \theta}}{\sin \theta H_{\frac{n \pi}{\kappa}}^{\prime}\left(k \sin \theta r_{S}\right)} .
$$

\section{References}

[1] H. F. Olson, Elements of Acoustical Engineering, 2nd Edition, D. Van Nostrand Company, Inc., 120 Alexander St., Princeton, New Jersey, 1957.

[2] A. Berkhout, D. D. Vries, P. Vogel, Acoustic control by wave field synthesis, J. Acoust. Soc. of Am. 93 (1993) 2764-2778. doi:10.1121/1.405852.

[3] J. Daniel, S. Moreau, R. Nicol, Further investigations of highorder ambisonics and wavefield synthesis for holophonic sound imaging, in: 114th Convention of the Audio Engineering Society, Amsterdam, The Netherlands, 2003.

[4] M. Poletti, Three-dimensional surround sound systems based on spherical harmonics, J. Audio Eng. Soc. 53 (11) (2005) 10041025 .

[5] J. Ahrens, S. Spors, An analytical approach to sound field reproduction using circular and spherical loudspeaker distributions, Acta Acustica united with Acustica 94 (6) (2008) 988-999. doi: 10.3813/AAA. 918115 .

[6] M. Kolundzija, C. Faller, M. Vetterli, Baffled circular loudspeaker array with broadband high directivity, in: Proceedings of the IEEE International Conference on Acoustics, Speech, and Signal Processing, ICASSP 2010, 14-19 March 2010, Sheraton Dallas Hotel, Dallas, Texas, USA, 2010, pp. 73-76. doi:10.1109/ ICASSP. 2010.5496204.

[7] M. Poletti, T. Betlehem, Design of a prototype variable directivity loudspeaker for improved surround sound reproduction in rooms, in: AES 52nd International Conference: Sound Field Control - Engineering and Perception, Guildford, UK, 2013. URL http://www . aes .org/e-lib/browse. cfm?elib=16926

[8] M. A. Poletti, T. Betlehem, T. D. Abhayapala, Higher-order loudspeakers and active compensation for improved $2 \mathrm{~d}$ sound field reproduction in rooms, J. Audio Eng. Soc. 63 (1/2) (2015) 31-45.

URL http://www.aes.org/e-lib/browse.cfm?elib=17564

[9] S. Spors, R. Rabenstein, J. Ahrens, The theory of wave field synthesis revisited, in: Proc. 124th Convention of the Audio Engineering Society, Amsterdam, The Netherlands, 2008.
[10] J. Ahrens, H. Wierstorf, S. Spors, Comparison of higher order ambisonics and wave field synthesis with respect to spatial discretization artifacts in time domain, in: AES 40th International Conference: Spatial Audio: Sense the Sound of Space, Tokyo, Japan, 2010.

URL http://www . aes.org/e-lib/browse. cfm?elib=15563

[11] F. M. Fazi, Sound field reproduction, Ph.D. thesis, University of Southampton, Faculty of Engineering, Science and Mathematics, Institute of Sound and Vibration Research (2010).

[12] J. Ahrens, Analytic Methods of Sound Field Synthesis, T-Labs Series in Telecommunication Services, Springer-Verlag, Berlin, Germany, 2012. doi:10.1007/978-3-642-25743-8.

[13] F. Zotter, A. Sontacchi, R. Höldrich, Modeling a spherical loudspeaker system as multipole source, Fortschritte der Akustik DAGA, Stuttgart, Germany 33 (1) (2007) 221.

[14] M. Møller, M. Olsen, F. T. Agerkvist, J. Dyreby, G. K. Munch, Circular loudspeaker arrays with controllable directivity, in: 128th Audio Engineering Society Convention, London, UK, 2010. URL http://www.aes.org/e-lib/browse. cfm?elib=15309

[15] M. Kolundzija, C. Faller, M. Vetterli, Design of a compact cylindrical loudspeaker array for spatial sound reproduction, in: 130th Convention of the Audio Engineering Society, Audio Engineering Society, London, UK, 2011.

[16] M. Shin, F. M. Fazi, P. A. Nelson, F. C. Hirono, Controlled sound field with a dual layer loudspeaker array, J. Sound and Vib. 333 (16) (2014) 3794-3817. doi:10.1016/j.jsv.2014.03.025.

[17] P. Coleman, P. J. Jackson, M. Olik, M. Olsen, M. Moller, J. A. Pedersen, The influence of regularization on anechoic performance and robustness of sound zone methods, in: Proceedings for the 21st International Congress on Acoustics, Montréal, Canada, Vol. 19, ASA, 2013, p. 055055. doi:10.1121/1.4799031.

[18] F. M. Fazi, M. Shin, F. Olivieri, S. Fontana, Y. Lang, Comparison of pressure-matching and mode-matching beamforming for methods for circular loudspeaker arrays, in: 137th Convention of the Audio Engineering Society, Los Angeles, USA, 2014.

[19] F. M. Fazi, M. Shin, F. Olivieri, S. Fontana, Low frequency performance of circular loudspeaker arrays, in: 138th Convention of the Audio Engineering Society, Audio Engineering Society, Warsaw, Poland, 2015.

[20] A. Farina, L. Chiesi, Measuring spatial mimo impulse responses in rooms employing spherical transducer arrays, in: AES International Conference on Sound Field Control - Engineering and Perception, Guildford, UK, 2016.

[21] E. G. Williams, Fourier Acoustic: Sound Radiation and Nearfield Acoustical Holography, Academic Press, 24-28 Oval Road, London NW1 7DX, UK, 1999.

[22] J. Meyer, Beamforming for a circular microphone array mounted on spherically shaped objects, J. Acoust. Soc. Am. 109 (1) (2001) 185-193. doi:10.1121/1.1329616.

[23] J. Meyer, G. W. Elko, A highly scalable spherical microphone array based on an orthonormal decomposition of the soundfield, in: 2002 IEEE International Conference on Acoustics, Speech, and Signal Processing, Orlando, Florida, USA, IEEE, 2002, pp. 1781-1784. doi:10.1109/ICASSP. 2002.5744968.

[24] B. Rafaely, Analysis and design of spherical microphone arrays, IEEE Trans. Speech and Audio Process. 13 (1) (2005) 135-143. doi:10.1109/TSA.2004.839244.

[25] D. N. Zotkin, R. Duraiswami, N. A. Gumerov, Plane-wave decomposition of acoustical scenes via spherical and cylindrical microphone arrays, IEEE Trans. Audio, Speech, and Lang. Process. 18 (1) (2010) 2-16. doi:10.1109/TASL. 2009.2022000.

[26] T. Betlehem, M. A. Poletti, Two dimensional sound field reproduction using higher order sources to exploit room reflections, J. Acoust. Soc. Am. 135 (4) (2014) 1820-1833. doi:http: //dx.doi.org/10.1121/1.4868376.

[27] T. Betlehem, W. Zhang, M. A. Poletti, T. D. Abhayapala, Personal sound zones: Delivering interface-free audio to multiple listeners, IEEE Signal Process. Magazine 32 (2) (2015) 81-91. doi:10.1109/msp.2014.2360707.

[28] H. Pomberger, F. Zotter, Modal sound field decomposition applicable for a limited range of directions, in: A. Peretti, J. Scheuren 
(Eds.), Fortschritte der Akustik - AIA-DAGA, full paper Edition, Berlin, Germany, 2013, procedure: without peer reviewing.

[29] H. Pomberger, F. Pausch, Design and evaluation of a spherical segment array with double cone, Acta Acustica united with Acustica 100 (5) (2014) 921-927. doi:10.3813/aaa.918771.

[30] F.-M. Hoffmann, F. M. Fazi, S. Fontana, Sound field control with hemi-cylindrical loudspeaker arrays, in: Audio Engineering Society Conference: 2016 AES International Conference on Sound Field Control, Guildford, UK, 2016.

URL http://www. aes.org/e-lib/browse. cfm?elib=18322

[31] J. Asvestas, J. Bowman, P. Christiansen, O. Einarsson, R. Kleinman, D. Sengupta, T. Senior, F. Sleator, P. Uslenghi, N. Zitron, Electromagnetic and Acoustic Scattering by Simple Shapes, North-Holland Pub. Co., Amsterdam, Netherlands, 1970.

URL https://books . google.co.uk/books?id=ueENAQAAIAAJ

[32] M. Buckingham, Acoustic propagation in a wedge-shaped ocean with perfectly reflecting boundaries, in: L. Felsen (Ed.), Hybrid Formulation of Wave Propagation and Scattering, Springer, Dordrecht, Netherlands, 1984, pp. 77-99. doi: 10.1007/978-94-009-6219-4_6.

[33] W. Luo, R. Zhang, Exact solution of three-dimensional acoustic field in a wedge with perfectly reflecting boundaries, Science China Physics, Mechanics \& Astronomy 58 (9) (2015) 1-10. doi:10.1007/s11433-015-5691-6.

[34] F.-M. Hoffmann, F. M. Fazi, E. G. Williams, S. Fontana, A general radiation model for sound fields and nearfield acoustical holography in wedge propagation spaces, J. Acoust. Soc. Am. 142 (3) (2017) 1249-1260. doi:10.1121/1.4998573.

[35] E. Kreyszig, Introductory Functional Analysis with Application, John Wiley \& Sons, New York, USA, 1978.

[36] J. Blauert, N. Xiang, Acoustics for Engineers, Springer, Berlin, Germany, 2008. doi:10.1007/978-3-540-76348-2.

[37] N. Ahmed, T. Natarajan, K. R. Rao, Discrete cosine transform, IEEE Trans. on Computers C-23 (1) (1974) 90-93. doi:10.1109/ T-C. 1974.223784.

[38] Z. Li, R. Duraiswami, E. Grassi, L. S. Davis, Flexible layout and optimal cancellation of the orthonormality error for spherical microphone arrays, in: 2004 IEEE International Conference on Acoustics, Speech, and Signal Processing, Montréal, Canada, 2004, pp. 1520-6149. doi:10.1109/ICASSP. 2004.1326758.

[39] S. Spors, R. Rabenstein, Spatial aliasing artifacts produced by linear and circular loudspeaker arrays used for wave field synthesis, in: 120th Convention of the Audio Engineering Society, Paris, France, 2006.

[40] B. Rafaely, B. Weiss, E. Bachmat, Spatial aliasing in spherical microphone arrays, IEEE Trans. Signal Process. 55 (2007) 10031010. doi:10.1109/TSP. 2006.888896.

[41] D. L. Alon, B. Rafaely, Spherical microphone array with optimal aliasing cancellation, in: Proceedings of the 27th Convention of Electrical and Electronics Engineering in Israel (IEEE), Eilat, Israel, 2012, pp. $1-5$.

[42] D. L. Alon, B. Rafaely, Spatial aliasing-cancellation for circular microphone arrays, in: 4th Joint Workshop on Hands-free Speech Communication and Microphone Arrays (HSCMA), Nancy, France, 2014, pp. 137-141. doi:10.1109/HSCMA.2014.6843267.

[43] D. B. Ward, T. D. Abhayapala, Reproduction of a plane-wave sound field using an array of loudspeakers, IEEE Trans. Speech and Audio Process. 9 (6) (2001) 697-707. doi:10.1109/89. 943347.

[44] M. A. Poletti, A unified theory of horizontal holographic sound systems, J. Audio Eng. Soc. 48 (12) (2000) 1155-1182. URL http://www.aes.org/e-lib/browse.cfm?elib $=12033$ 